\title{
NUMERICAL SIMULATION OF BLOOD FLOWS THROUGH A POROUS INTERFACE
}

\author{
Miguel A. Fernández ${ }^{1}$, Jean-Frédéric Gerbeau ${ }^{1}$ And Vincent Martin ${ }^{2}$
}

\begin{abstract}
We propose a model for a medical device, called a stent, designed for the treatment of cerebral aneurysms. The stent consists of a grid, immersed in the blood flow and located at the inlet of the aneurysm. It aims at promoting a clot within the aneurysm. The blood flow is modelled by the incompressible Navier-Stokes equations and the stent by a dissipative surface term. We propose a stabilized finite element method for this model and we analyse its convergence in the case of the Stokes equations. We present numerical results for academical test cases, and on a realistic aneurysm obtained from medical imaging.
\end{abstract}

Mathematics Subject Classification. 65M60, 74K25, 76D05, 76Z05.

Received February 5, 2007.

Published online August 12, 2008.

\section{INTRODUCTION}

This work is motivated by the numerical simulation of a new medical device ${ }^{3}$ designed for the treatment of cerebral aneurysms located on bifurcations of arteries. This device consists of a wire metal mesh tube, called a stent. Contrary to the usual stents - which are typically used to keep arteries open and which are located on the vessel wall - this stent is immersed in the blood flow (Fig. 1). The purpose of this device is to reduce the flux within the aneurysm in order to occlude it by a clot. For practical reasons, a portion of the stent is also present in front of collateral arteries, with a risk of adverse effect in the blood flows. The motivations of modelling are, first, quantify the desired reduction of vorticity and shear stress in the aneurysms and, second, the non-desired reduction of blood flows in collateral branches.

For reasons that will be developed in Section 1, the geometrical details of the stent wires are ignored in this study. Our model thus consists in an homogenized porous interface immersed in the flow. From the mathematical standpoint, the flow is assumed to be governed by the incompressible Navier-Stokes equations and the stent is modelled by a dissipative surface term added to the left-hand side of the equations.

This additional dissipative term induces a jump of the stress across the stent surface which may raise numerical issues. On the one hand, continuous approximations of the pressure may give very inaccurate results, on the other hand, discontinuous approximations of the pressure typically lead to expensive simulations. To circumvent

\footnotetext{
Keywords and phrases. Stabilized finite element, sieve problem, blood flow, terminal aneurysm, stent, fluid-structure interaction.

${ }^{1}$ INRIA Paris-Rocquencourt, BP 105, 78153 Le Chesnay, France. Miguel.Fernandez@inria.fr;

${ }^{2}$ University of Technology of Compiègne, LMAC, GI, Royallieu, BP 20529, 60205 Compiègne, France. Vincent.Martin@utc.fr

${ }^{3}$ The LyLyk ${ }^{\circledR}$ device, by Cardiatis.
} 


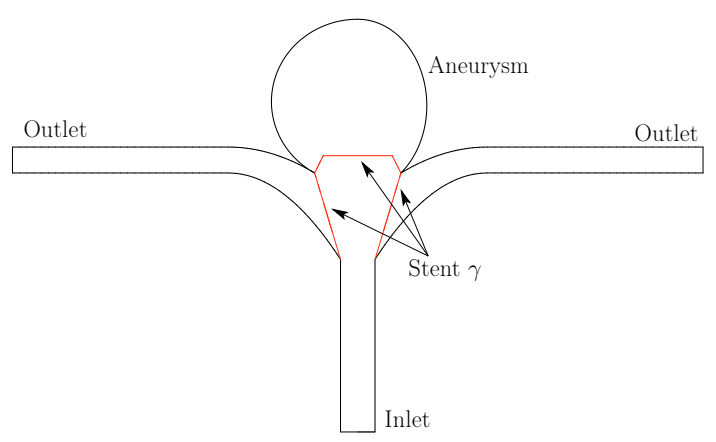

FIGURE 1. Example of a two-dimensional domain $\Omega$ with a stent ("LyLyk" device by Cardiatis) represented by a line $\gamma$. The part of the stent that lies on the arterial wall (vertical artery) is not represented.

these issues, we propose to use stabilized finite elements, with continuous pressure, such as $P_{1} / P_{1}$ elements, and to introduce a "fissure" in the mesh on the stent surface, in order to allow for the pressure to be discontinuous at the interface only. The proposed stabilization formulation is made of two contributions: a standard residual based stabilization giving a $L^{2}$-control on the pressure gradient; an interface based stabilization providing a $L^{2}$-control on the jump of the pressure. In Section 2, a complete convergence analysis and an optimal error estimate are presented for the Stokes equations.

Numerical results are presented in Section 3. The theoretical convergence rate of Section 2 is confirmed on an academical example where the solution is known in a closed-form. We also propose a validation test on a simple two dimensional configuration. We end the paper with results on realistic three dimensional geometries obtained from medical imaging. In that case, we also take into account the fluid-structure interaction of the blood with the wall artery.

\section{Motivations And modelling}

The purpose of the stent considered in this work is to treat intra-cranial terminal aneurysm located at an artery bifurcation (see e.g. [25]). Contrary to the stents commonly used to treat stenoses or side aneurysms, this stent is closed at one end. Moreover, it is characterized by very thin wires (40 $\mu \mathrm{m})$, very small windows $(100 \mu \mathrm{m})$ and a multilayer structure. It has the shape of a small, finely woven metallic socket, whose tip is intended to be inserted inside the aneurysm, while the sleeve is in contact with the main artery. The part inside the aneurysm is finely braided, in order to reduce blood flow, and hopefully cause a thrombosis inside the aneurysm. The lateral part is more coarsely braided, in order to let the blood flow into the two daughter arteries.

In general, in finite element studies of stents in blood flows, each wire of the stent is meshed (see for instance $[2,26]$ ). This approach requires an important work to generate the mesh, and is computationally heavy. Its interest is to provide a precise description of the local flow alterations caused by the stent. In our case, this approach is very expensive due to the very small windows of the stent and the complex multilayer structure. Moreover from the modelling viewpoint, it would be questionable to claim that one solves a resolution of $40 \mu \mathrm{m}$ while neglecting the red blood cells ( $8 \mu \mathrm{m}$ diameter) which occupy about $50 \%$ of the blood volume. This is why we preferred to model the stent "macroscopically" by a mean porous surface immersed in the flow. Note that 


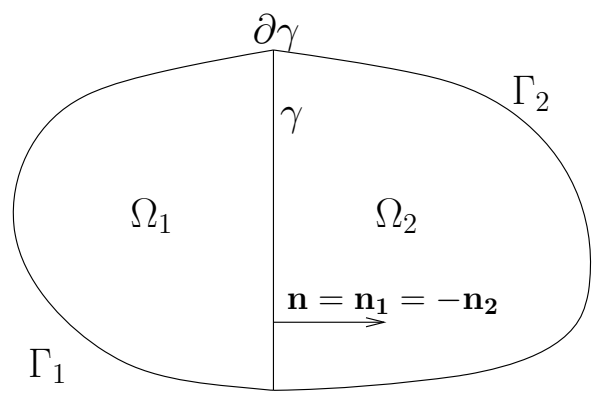

Figure 2. The domain $\Omega$ decomposed into two subdomains $\Omega_{1}$ and $\Omega_{2}$, separated by the stent $\gamma$.

we neglect any other physical aspects of the stent: deformation of its structure, interaction with the vessel wall, mechanical stability of the stent in the artery, etc.

Let $\Omega$ be a simply connected smooth domain in $\mathbb{R}^{n}, n=2$ or 3 , and $\Gamma=\partial \Omega$ its regular boundary. We suppose (see Fig. 1) that the stent can be represented by a regular surface $\gamma$ immersed in $\Omega$.

Let $\mathbf{u}$ and $p$ be the velocity and the pressure of the fluid. The strain rate tensor $\mathbf{D}$ and the Cauchy stress tensor $\mathbf{T}$ associated with $\mathbf{u}, p$ are defined by

$$
\mathbf{D}(\mathbf{u})=\frac{1}{2}\left(\nabla \mathbf{u}+\nabla \mathbf{u}^{\top}\right) \text { and } \mathbf{T}(\mathbf{u}, p)=-p \mathbf{I}+2 \mu \mathbf{D}(\mathbf{u})
$$

where $\mu$ is the dynamic viscosity of the fluid, $\mathbf{u}$ is the velocity, $p$ the pressure and $\mathbf{I}$ is the identity matrix in $\mathbb{R}^{n}$.

The blood is assumed to be governed by the incompressible Navier-Stokes equations. This is a commonly accepted hypothesis in the large vessels we are considering. To model the stent, we propose to add a dissipative surface term to the conservation of momentum equations. Thus, the model formally reads: find $\mathbf{u}$ and $p$ such that

$$
\begin{aligned}
& \rho_{f}\left(\frac{\partial \mathbf{u}}{\partial t}+\mathbf{u} \cdot \nabla \mathbf{u}\right)-\operatorname{div} \mathbf{T}(\mathbf{u}, p)+\mathbf{R}_{\gamma} \mathbf{u} \delta_{\gamma}=\mathbf{f} \quad \text { in } \Omega \\
& \operatorname{div} \mathbf{u}=0 \quad \text { in } \Omega,
\end{aligned}
$$

where $\rho_{f}$ is the density of the fluid, and $\mathbf{f}$ is the body force density, $\mathbf{R}_{\gamma}$ is a symmetric and positive definite tensor that represents the dissipation due to the stent, and $\delta_{\gamma}$ is the Dirac measure on the stent surface $\gamma$. In other words, for $\mathbf{v} \in\left[H^{1}(\Omega)\right]^{n}$,

$$
\left\langle\mathbf{R}_{\gamma} \mathbf{u} \delta_{\gamma}, \mathbf{v}\right\rangle=\int_{\gamma} \mathbf{R}_{\gamma} \mathbf{u} \cdot \mathbf{v} \mathrm{d} \gamma
$$

A comment on this model is in order: the 3D Navier-Stokes equations give a precise information on local scales while the stent is governed by a rather rough model. This discrepancy may be considered as a weakness of the model. We nevertheless believe that our choice, in spite of its obvious limitations, may be relevant: on the one hand, we indeed need the local information provided by the Navier-Stokes equations (e.g. to evaluate the wall shear stress which is known to be important in hemodynamics), on the other hand, we cannot afford to resolve a space resolution of a $40 \mu \mathrm{m}$ wire and we are mainly interested in global effects induced by the presence of the stent (e.g. vorticity reduction in the aneurysm, flux modification in the branches).

\section{Numerical ANAlysis in the CASE OF StOKES Flows}

In this section, to simplify the numerical analysis and to explain how to compute the permeability tensor from homogenization results, we make two important assumptions: first we assume the fluid to be governed by the stationary Stokes equations; second we suppose that the stent is represented by a hyperplane $\gamma$ (see Fig. 2) 
that divides the domain $\Omega$ into two connected subdomains

$$
\Omega_{f}=\Omega \backslash \gamma=\Omega_{1} \cup \Omega_{2}, \quad \Omega_{1} \cap \Omega_{2}=\emptyset .
$$

We denote by $\mathbf{n}_{i}$ the outward normal on $\gamma$ viewed as a part of the $\Omega_{i}$ boundary, for $i=1,2$. We denote by $\Gamma_{i}$ the part of the boundary of $\Omega_{i}$ in common with the boundary of $\Omega$ :

$$
\Gamma_{i}=\partial \Omega_{i} \cap \Gamma, \quad i=1,2 .
$$

We also introduce

$$
\mathbf{u}_{i}=\left.\mathbf{u}\right|_{\Omega_{i}}, \quad p_{i}=\left.p\right|_{\Omega_{i}}, \quad \mathbf{f}_{i}=\left.\mathbf{f}\right|_{\Omega_{i}}, \quad \text { for } i=1,2 .
$$

We will consider the usual Sobolev spaces $H^{m}(\mathcal{O}), m \geq 0$, for a given bounded open set $\mathcal{O} \subset \mathbb{R}^{d}, 1 \leq d \leq n$. In particular, we have $L^{2}(\mathcal{O})=H^{0}(\mathcal{O})$. The scalar product in $L^{2}(\mathcal{O})$ is denoted by $(\cdot, \cdot)_{\mathcal{O}}$ and its norm by $\|\cdot\|_{0, \mathcal{O}}$. The closed subspaces $H_{0}^{1}(\mathcal{O})$, consisting of functions in $H^{1}(\mathcal{O})$ with zero trace on $\partial \mathcal{O}$, and $L_{0}^{2}(\mathcal{O})$, consisting of function in $L^{2}(\mathcal{O})$ with zero mean in $\mathcal{O}$, will also be used. We define the following notations:

$$
M=L^{2}(\Omega), \quad \tilde{M}=L_{0}^{2}(\Omega), \quad \mathbf{V}=\left[H_{0}^{1}(\Omega)\right]^{n},
$$

equipped with their usual norms $\|\cdot\|_{0, \Omega}$ and $\|\cdot\|_{1, \Omega}$. The subscript $\Omega$ will in general be omitted, when the norm is taken over the whole domain $\Omega$. We assume that the right-hand side $\mathbf{f} \in\left[L^{2}(\Omega)\right]^{n}$.

\subsection{Problem setting}

The Stokes counterpart of problem (1.1) reads in a two-domain formulation:

$$
\begin{aligned}
-\mu \Delta \mathbf{u}_{i}+\nabla p_{i} & =\mathbf{f}_{i} & & \text { in } \Omega_{i}, \quad i=1,2, \\
\operatorname{div} \mathbf{u}_{i} & =0 & & \text { in } \Omega_{i}, \quad i=1,2, \\
\mathbf{u}_{1} & =\mathbf{u}_{2} & & \text { on } \gamma, \\
\mu \nabla \mathbf{u}_{1} \cdot \mathbf{n}_{1}-p_{1} \mathbf{n}_{1}+\mu \nabla \mathbf{u}_{2} \cdot \mathbf{n}_{2}-p_{2} \mathbf{n}_{2} & =-\mathbf{R}_{\gamma} \mathbf{u} & & \text { on } \gamma, \\
\mathbf{u}_{i} & =0 & & \text { on } \Gamma_{i}, \quad i=1,2,
\end{aligned}
$$

where homogeneous Dirichlet boundary conditions on $\Gamma_{i}, i=1,2$ have been chosen for simplicity. Let $\mathbf{v}$ in $\mathbf{V}$ and $\mathbf{v}_{i}=\left.\mathbf{v}\right|_{\Omega_{i}}, i=1,2$. Multiplying the first equations of (2.1) by $\mathbf{v}_{i}, i=1,2$, integrating by parts and adding the results, we readily obtain

$$
\sum_{i=1}^{2}\left[\left(\mu \nabla \mathbf{u}_{i}, \nabla \mathbf{v}_{i}\right)_{\Omega_{i}}-\left(p_{i}, \operatorname{div} \mathbf{v}_{i}\right)_{\Omega_{i}}-\left(\mu \nabla \mathbf{u}_{i} \cdot \mathbf{n}_{i}-p_{i} \mathbf{n}_{i}, \mathbf{v}_{i}\right)_{\gamma}\right]=(\mathbf{f}, \mathbf{v})_{\Omega} .
$$

Using (2.1) $)_{4}$ and the regularity of $\mathbf{u}$ and $\mathbf{v}$, we obtain a variational formulation of problem (2.1): find $\mathbf{u} \in \mathbf{V}$ and $p \in \tilde{M}$ such that

$$
\left\{\begin{aligned}
(\mu \nabla \mathbf{u}, \nabla \mathbf{v})_{\Omega}+\left(\mathbf{R}_{\gamma} \mathbf{u}, \mathbf{v}\right)_{\gamma}-(\operatorname{div} \mathbf{v}, p)_{\Omega} & =(\mathbf{f}, \mathbf{v})_{\Omega} & & \forall \mathbf{v} \in \mathbf{V} \\
(\operatorname{div} \mathbf{u}, r)_{\Omega} & =0 & & \forall r \in \tilde{M}
\end{aligned}\right.
$$

With the above assumptions on the data, we can state the following result.

Proposition 2.1. Problem (2.3) has a unique solution.

Proof. We define the bilinear form $a(\cdot, \cdot)$ on $\left[H_{0}^{1}(\Omega)\right]^{n} \times\left[H_{0}^{1}(\Omega)\right]^{n}$ by

$$
a(\mathbf{u}, \mathbf{v})=(\mu \nabla \mathbf{u}, \nabla \mathbf{v})_{\Omega}+\left(\mathbf{R}_{\gamma} \mathbf{u}, \mathbf{v}\right)_{\gamma}
$$




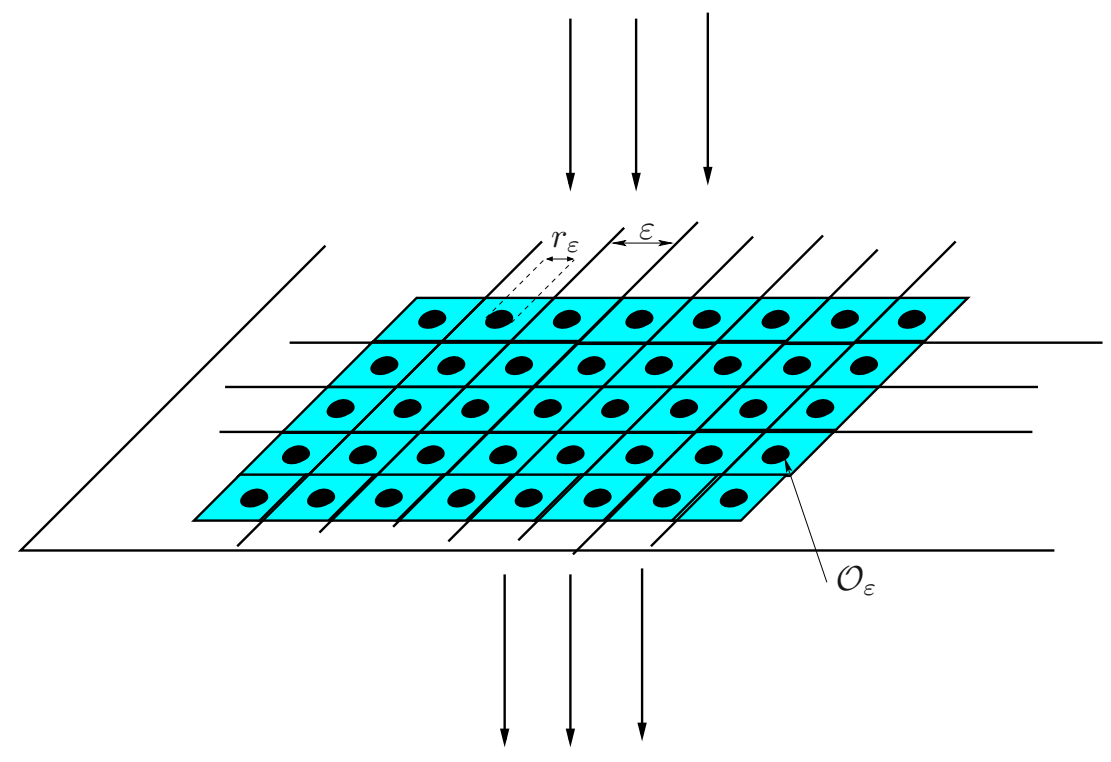

FiguRE 3. Planar Dirichlet sieve: regularly spaced obstacles where the flow is zero.

This bilinear form is continuous (consequence of the trace theorem) and coercive (consequence of the Poincaré inequality and the positiveness of $\mathbf{R}_{\gamma}$ ). This result is therefore a direct consequence of the standard theory of the Stokes equations (see [19]).

\subsection{Microstructure and non-scalar resistivity tensor}

The heuristic model proposed above can be rigorously justified in some cases. Moreover, an explicit form of the resistivity tensor $\mathbf{R}_{\gamma}$ can be obtained from extra computations, thus linking the microstructure of the sieve to its macroscopic impact. In this work, we did not use directly these results since we based our resistance tensor on simpler experimental data. Nevertheless, we find interesting to give some ideas about the non-scalar tensors that could be derived from a known microscopic structure.

Consider the 3D Stokes flow through a planar sieve made of a set of periodically spaced 2D identical obstacles and assume no-slip boundary conditions on the obstacles (see Fig. 3). Then, three main asymptotic behaviors can be observed depending upon the limit $\alpha=\lim _{\varepsilon \rightarrow 0}\left(r_{\varepsilon} / \varepsilon^{2}\right)$ of the ratio of the radius $r_{\varepsilon}$ of the obstacles over the square of the spatial periodicity $\varepsilon$ :

- when $\alpha=0$, the obstacles are asymptotically not seen by the flow: the limit problem is the Stokes problem in the whole domain;

- when $\alpha=+\infty$, the obstacles become asymptotically a wall, and the limit problem consists of two independent Stokes problems with no-slip conditions on the interface;

- when $0<\alpha<+\infty$, the limit problem is precisely (2.1).

In that last case, the resistivity tensor $\mathbf{R}_{\gamma}$ can be computed from the following cell-problem. Let $\mathcal{O}$ be the unit obstacle in $[-1,1] \times[-1,1]$. We denote by $\mathbf{e}_{k}$ the $k$-th canonical vector of $\mathbb{R}^{3}$ and we define $\left(\mathbf{w}^{k}, q^{k}\right)$, for $k=1,2,3$, as the solution of the Stokes problem:

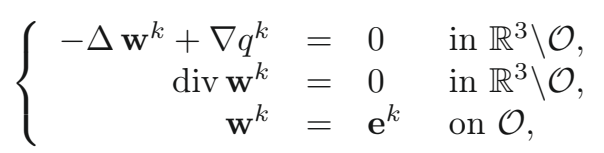


with $\lim _{|y| \rightarrow \infty} \mathbf{w}^{k}(y)=0, \lim _{|y| \rightarrow \infty} \nabla \mathbf{w}^{k}(y)=0$ and $\lim _{|y| \rightarrow \infty} q^{k}(y)=0$. Then the permeability tensor is given by:

$$
\mathbf{R}_{\gamma}=\frac{\alpha \mu}{4}\left[\int_{\mathbb{R}^{3} \backslash \mathcal{O}} \nabla \mathbf{w}^{k} \cdot \nabla \mathbf{w}^{l} \mathrm{~d} y\right]_{k, l=1,2,3} .
$$

It can be proved that $\mathbf{R}_{\gamma}$ is symmetric and positive definite. The proofs of these statements can be found in $[1,4]$. We also refer the reader to $[10,11,23]$.

\subsection{Stabilized finite element approximation}

The classical Galerkin method applied to (2.3) requires the fulfillment of a inf-sup condition [19] between the velocity and pressure spaces, which leads to formulations involving mixed interpolations [3]. From the computational point of view, it is more convenient to deal with equal order velocity-pressure interpolations, but in that case, stability has to be enforced in another way. A standard approach consists in using stabilized finite element methods where some terms are added to the standard Galerkin formulation in order to enhance the stability of the method (see e.g. $[5,9,20,28]$ ). For instance, in $[20,28]$ stability in achieved by adding a residual based term which gives $L^{2}$-control of the gradient of the pressure. Optimal error estimates, for arbitrary polynomial order $k \geq 1$, can be derived assuming that the solution is smooth enough, typically $(\mathbf{u}, p) \in$ $\left[H^{k+1}(\Omega)\right]^{n} \times H^{k}(\Omega)$. In this work, we extend this approach to our specific situation.

It is important to notice that, due to the presence of the interface resistive term, one cannot expect more than $(\mathbf{u}, p) \in\left[H^{1}(\Omega)\right]^{n} \times L^{2}(\Omega)$ as global regularity of the solution of (2.3). As a result, some additional cares have to be taken in order to approximate (2.3) by finite elements. On the other hand it seems reasonable to assume that

$$
\left(\mathbf{u}_{i}, p_{i}\right) \in\left[H^{k+1}\left(\Omega_{i}\right)\right]^{n} \times H^{k}\left(\Omega_{i}\right), \quad i=1,2 .
$$

In this section, we propose a conforming stabilized finite element method, that allows equal order interpolations, and for which we can prove optimal error estimates under this reduced regularity. The key ingredient consists in combining the techniques of $[20,28]$ with an interface based stabilization allowing pressure discontinuities through the interface $\gamma$.

\subsubsection{Preliminaries}

In what follows, we shall assume $\Omega$ to be a Lipschitz-continuous domain in $\mathbb{R}^{n}(n=2$ or 3$)$ with a polyhedral boundary $\partial \Omega$ and outward pointing normal $\mathbf{n}$. We shall also assume the viscosity $\mu$ to be constant. Let $\left\{\mathcal{T}_{h}\right\}_{0<h \leq 1}$ denotes a regular family of triangulations of the domain $\Omega$ (in the sense of [7]). For each triangulation $\mathcal{T}_{h}$, the subscript $h \in(0,1]$ refers to the level of refinement of the triangulation, which is defined by

$$
h=\max _{T \in \mathcal{T}_{h}} h_{T}
$$

with $h_{T}$, the diameter of $T$. We shall assume for the sake of simplicity the quasi-uniformity of the triangulation, i.e. there exist two positive constants $C_{\min }$ and $C_{\max }$ such that

$$
C_{\min } h_{T} \leq h \leq C_{\max } h_{T}, \quad \forall T \in \mathcal{T}_{h} .
$$

Moreover, for all $0<h \leq 1$, the triangulation $\mathcal{T}_{h}$ is supposed to be conforming with the interface $\gamma$. Let $\mathcal{G}_{h}$ be the set of inter-element boundaries of $\mathcal{T}_{h}$ (faces in 3D, edges in 2D) lying on $\gamma$. For a given piecewise continuous function $\varphi$, the jump $\llbracket \varphi \mathbf{n} \rrbracket$ over an edge $E \in \mathcal{G}_{h}$ is defined by

$$
\llbracket \varphi \mathbf{n} \rrbracket(\mathbf{x})=\lim _{t \rightarrow 0^{+}}\left(\varphi\left(\mathbf{x}+t \mathbf{n}_{E}^{1}\right) \mathbf{n}_{E}^{1}+\varphi\left(\mathbf{x}+t \mathbf{n}_{E}^{2}\right) \mathbf{n}_{E}^{2}\right),
$$

where $\mathbf{x} \in E$, and $\mathbf{n}_{E}^{1}$ (respectively $\mathbf{n}_{E}^{2}$ ) is the unit normal vector to $E$ pointing outward $\Omega_{1}$ (respectively outward $\Omega_{2}$ ). 
We consider equal order approximations of order $k \geq 1$ for the velocity and the pressure. Both velocity and pressure approximations will be continuous at inter-element boundaries, except for the pressure that will be discontinuous on the faces in 3D (on the edges in 2D) of the interface $\gamma$. Thus, we introduce the following velocity and pressure discrete spaces:

$$
\begin{aligned}
\mathbf{V}_{h}^{k} & =\left\{\mathbf{v}_{h} \in \mathcal{C}^{0}(\bar{\Omega}):\left.\forall T \in \mathcal{T}_{h} \mathbf{v}_{h}\right|_{T} \in\left(P_{k}\right)^{n}\right\} \cap \mathbf{V} \\
M_{h}^{k} & =\left\{q_{h} \in \tilde{M}:\left.p\right|_{\Omega_{i}} \in \mathcal{C}^{0}\left(\bar{\Omega}_{i}\right), i=1,2,\left.\quad \forall T \in \mathcal{T}_{h} q_{h}\right|_{T} \in P_{k}\right\}
\end{aligned}
$$

Finally, for $i=1,2$, let $M_{h, i}^{k}$ the space of the restrictions of elements of $M_{h}^{k}$ to $\Omega_{i}$,

$$
M_{h, i}^{k}=\left\{q_{h \mid \Omega_{i}}: q_{h} \in M_{h}^{k}\right\} .
$$

In order to approximate (non-smooth) functions of $\left[H_{0}^{1}(\Omega)\right]^{n}$ with $n \geq 2$, which are not in the domain of the Lagrange interpolation operator, we consider the Scott-Zhang interpolation operator $S Z_{h}^{k}$ onto $\mathbf{V}_{h}^{k}$ (see [24]). The following error estimate then holds (see [24] or [13], p. 70),

$$
\left\|\mathbf{u}-S Z_{h}^{k} \mathbf{u}\right\|_{0, \Omega} \leq c_{0} h\|\mathbf{u}\|_{1, \Omega}
$$

In particular, using a trace inequality (see [8,27]), we also have

$$
\left\|\mathbf{u}-S Z_{h}^{k} \mathbf{u}\right\|_{0, E} \leq C_{0} h^{1 / 2}\|\mathbf{u}\|_{1, \Omega}
$$

Let $I_{h}^{k}$ be the Lagrange interpolation operator onto $\mathbf{V}_{h}^{k}$. Thus, we have the following estimates (see [13]), for $T \in \mathcal{T}_{h}$, and $0 \leq m \leq 2,2 \leq l \leq k+1$ :

$$
\left\|\mathbf{u}-I_{h}^{k} \mathbf{u}\right\|_{m, T} \leq c_{1} h^{l-m}\|\mathbf{u}\|_{l, T} \quad \mathbf{u} \in\left[H^{l}(T)\right]^{n} .
$$

Analogously, using a trace inequality, for $E \in \mathcal{G}_{h}\left(E=T_{1} \cap T_{2}, T_{1}, T_{2} \in \mathcal{T}_{h}\right)$, we also have

$$
\left\|\mathbf{u}-I_{h}^{k} \mathbf{u}\right\|_{0, E} \leq\left. c_{2} h^{l-1 / 2} \sum_{i=1,2}\|\mathbf{u}\|_{l, T_{i}} \quad \mathbf{u}\right|_{T_{i}} \in\left[H^{l}\left(T_{i}\right)\right]^{n}, i=1,2, \mathbf{u} \in\left[H^{1}\left(T_{1} \cup T_{2}\right)\right]^{n} .
$$

For $i=1,2$ let $J_{h, i}^{k}: L^{2}\left(\Omega_{i}\right) \mapsto M_{h, i}^{k}$ be the $L^{2}$-projection onto $M_{h, i}^{k}$. Thus, for $0 \leq m \leq 1,1 \leq l \leq k+1$, we have

$$
\begin{aligned}
\left\|p_{i}-J_{h, i} p_{i}\right\|_{m, \Omega_{i}} & \leq c_{3} h^{l-m}\left\|p_{i}\right\|_{l, \Omega_{i}} \quad p_{i} \in H^{l}\left(\Omega_{i}\right), \\
\left\|p_{i}-J_{h, i} p_{i}\right\|_{0, \gamma} & \leq c_{4} h^{l-1 / 2}\left\|p_{i}\right\|_{l, \Omega_{i}} \quad p_{i} \in H^{l}\left(\Omega_{i}\right) .
\end{aligned}
$$

We then introduce the (global) operator $J_{h}^{k}: L^{2}(\Omega) \mapsto M_{h}^{k}$, defined by

$$
\left.\left(J_{h}^{k} p\right)\right|_{\Omega_{i}}=J_{h, i}^{k}\left(\left.p\right|_{\Omega_{i}}\right), \quad i=1,2 .
$$

From (2.10), it then follows that for $0 \leq m \leq 1,1 \leq l \leq k+1$,

$$
\begin{aligned}
& \left\|p-J_{h}^{k} p\right\|_{m, \Omega_{i}} \leq c_{3} h^{l-m}\|p\|_{l, \Omega_{i}} \quad p \in L^{2}(\Omega),\left.p\right|_{\Omega_{i}} \in H^{l}\left(\Omega_{i}\right), i=1,2, \\
& \left\|\llbracket p-J_{h}^{k} p \rrbracket\right\|_{0, \gamma} \leq c_{4} h^{l-1 / 2} \sum_{i=1,2}\|p\|_{l, \Omega_{i}} \quad p \in L^{2}(\Omega),\left.p\right|_{\Omega_{i}} \in H^{l}\left(\Omega_{i}\right), i=1,2 .
\end{aligned}
$$

Using the quasi-uniformity of the mesh, the following inverse and trace estimates hold (see $[8,13]$ ),

$$
\begin{gathered}
\left\|\Delta \mathbf{v}_{h}\right\|_{0, T} \leq c_{5} h^{-1}\left\|\nabla \mathbf{v}_{h}\right\|_{0, T} \quad \mathbf{v}_{h} \in \mathbf{V}_{h}^{k}, \\
\left\|\llbracket \nabla \mathbf{v}_{h} \cdot \mathbf{n} \rrbracket\right\|_{0, E} \leq c_{6} h^{-1 / 2}\left\|\mathbf{v}_{h}\right\|_{1, T_{1} \cup T_{2}} \quad \mathbf{v}_{h} \in \mathbf{V}_{h}^{k} .
\end{gathered}
$$


Finally, we also have the standard Sobolev trace inequality

$$
\left\|\left.\mathbf{u}\right|_{\gamma}\right\|_{0, \gamma} \leq c_{\gamma}\|\mathbf{u}\|_{1, \Omega} \quad \mathbf{u} \in \mathbf{V}
$$

\subsubsection{A stabilized finite element method}

Our finite element approximation for problem (2.3) reads: find $\left(\mathbf{u}_{h}, p_{h}\right) \in \mathbf{V}_{h}^{k} \times M_{h}^{k}$ such that

$$
B_{\delta, \mathbf{R}_{\gamma}}\left(\left(\mathbf{u}_{h}, p_{h}\right),\left(\mathbf{v}_{h}, q_{h}\right)\right)=F_{\delta}\left(\left(\mathbf{v}_{h}, q_{h}\right)\right), \quad \forall\left(\mathbf{v}_{h}, q_{h}\right) \in \mathbf{V}_{h}^{k} \times M_{h}^{k},
$$

with

$$
\begin{aligned}
B_{\delta, \mathbf{R}_{\gamma}}((\mathbf{u}, p),(\mathbf{v}, q))= & (\mu \nabla \mathbf{u}, \nabla \mathbf{v})_{\Omega}+\left(\mathbf{R}_{\gamma} \mathbf{u}, \mathbf{v}\right)_{\gamma}-(p, \operatorname{div} \mathbf{v})_{\Omega}+(q, \operatorname{div} \mathbf{u})_{\Omega} \\
& +\delta \sum_{T \in \mathcal{T}_{h}} \frac{h^{2}}{\mu}(-\mu \Delta \mathbf{u}+\nabla p, \nabla q)_{T} \\
& -\delta \sum_{E \in \mathcal{G}_{h}} \frac{h}{\mu}\left(\llbracket \mu \nabla \mathbf{u} \cdot \mathbf{n}-p \mathbf{n} \rrbracket+\mathbf{R}_{\gamma} \mathbf{u}, \llbracket q \mathbf{n} \rrbracket\right)_{E}, \\
F_{\delta}((\mathbf{v}, q))= & (\mathbf{f}, \mathbf{v})_{\Omega}+\delta \sum_{T \in \mathcal{T}_{h}} \frac{h^{2}}{\mu}(\mathbf{f}, \nabla q)_{T},
\end{aligned}
$$

and $\delta>0$ is a parameter independent of $h$ and that will be determined in Theorem 2.7.

The stabilization is made of two contributions: a residual based stabilization $[20,28]$ giving a $L^{2}$-control on the pressure gradient, and an interface based stabilization providing $L^{2}$-control on the jumps of the pressure. Both stabilizing terms seem necessary to establish an inf-sup condition independent of the discretization parameter (see Prop. 2.3).

The following proposition states the strong consistency of the discrete formulation (2.14).

Proposition 2.2. Assume that $(\mathbf{u}, p) \in\left[H^{1}(\Omega)\right]^{n} \times L^{2}(\Omega)$ and let $\left(\mathbf{u}_{h}, p_{h}\right) \in \mathbf{V}_{h}^{k} \times M_{h}^{k}$ be the solution of (2.14). Then

$$
B_{\delta, \mathbf{R}_{\gamma}}\left(\left(\mathbf{u}-\mathbf{u}_{h}, p-p_{h}\right),\left(\mathbf{v}_{h}, q_{h}\right)\right)=0, \quad \forall\left(\mathbf{v}_{h}, q_{h}\right) \in \mathbf{V}_{h}^{k} \times M_{h}^{k} .
$$

Proof. It is a direct consequence of the strong consistency of the standard Galerkin method, the $\gamma$-conformity of the triangulation $\mathcal{T}_{h}$ and the fact that the solution $(\mathbf{u}, p)$ satisfies

$$
\begin{aligned}
\mathbf{f}+\mu \Delta \mathbf{u}-\nabla p=0, & \text { in } \quad\left[\mathcal{D}^{\prime}\left(\Omega_{i}\right)\right]^{n}, \quad i=1,2, \\
\llbracket \mu \nabla \mathbf{u} \cdot \mathbf{n}-p \mathbf{n} \rrbracket_{\gamma}+\mathbf{R}_{\gamma} \mathbf{u}=0, & \text { in } \quad\left[H^{-1 / 2}(\gamma)\right]^{n} .
\end{aligned}
$$

In particular, notice that these expressions and the regularity of $\mathbf{f}$ yield

$$
\begin{aligned}
\mu \Delta \mathbf{u}-\nabla p \in\left[L^{2}(T)\right]^{n}, & \forall T \in \mathcal{T}_{h}, \\
\llbracket \mu \nabla \mathbf{u} \cdot \mathbf{n}-p \mathbf{n} \rrbracket_{E}+\mathbf{R}_{\gamma} \mathbf{u} \in\left[L^{2}(E)\right]^{n}, & \forall E \in \mathcal{G}_{h},
\end{aligned}
$$

so that the broken integrals in (2.15) are well defined.

\subsubsection{Stability}

In what follows we assume that there exist two positive constants $r_{\min }$ and $r_{\max }$ such that

$$
r_{\min }|\mathbf{y}|^{2} \leq \mathbf{y}^{\mathrm{T}} \mathbf{R}_{\gamma}(\mathbf{x}) \mathbf{y} \leq r_{\max }|\mathbf{y}|^{2}, \quad \forall \mathbf{x} \in \gamma, \quad \forall \mathbf{y} \in \mathbb{R}^{n}
$$


The convergence of the discrete solution will be stated in terms of the following mesh-dependent norm on $\mathbf{V}_{h}^{k} \times M_{h}^{k}:$

$$
\|(\mathbf{u}, p)\|_{h}^{2}=\left|\mu^{1 / 2} \mathbf{u}\right|_{1, \Omega}^{2}+\left\|r_{\min }^{1 / 2} \mathbf{u}\right\|_{0, \gamma}^{2}+\delta \sum_{T \in \mathcal{T}_{h}} \frac{h^{2}}{\mu}\|\nabla p\|_{0, T}^{2}+\delta \sum_{E \in \mathcal{G}_{h}} \frac{h}{\mu}\|\llbracket p \rrbracket\|_{0, E}^{2}+\left\|\mu^{-1 / 2} p\right\|_{0, \Omega}^{2},
$$

for all $(\mathbf{u}, p) \in \mathbf{V}_{h}^{k} \times M_{h}^{k}$.

The main stability result for our method is stated in the following theorem:

Theorem 2.3. Assume that

$$
0<\delta \leq \frac{1}{c_{5}^{2}+2 c_{6}^{2}}, \quad \delta h \leq \frac{\mu r_{\min }}{2 r_{\max }^{2}}
$$

Then, there exists a constant $\beta=\beta\left(\delta, r_{\min }, r_{\max }, \mu\right)>0$ such that

$$
\inf _{\left(\mathbf{u}_{h}, p_{h}\right) \in \mathbf{V}_{h}^{k} \times M_{h}^{k}} \sup _{\left(\mathbf{v}_{h}, q_{h}\right) \in \mathbf{V}_{h}^{k} \times M_{h}^{k}} \frac{B_{\delta, \mathbf{R}_{\gamma}}\left(\left(\mathbf{u}_{h}, p_{h}\right),\left(\mathbf{v}_{h}, q_{h}\right)\right)}{\left\|\left(\mathbf{u}_{h}, p_{h}\right)\right\|_{h}\left\|\left(\mathbf{v}_{h}, q_{h}\right)\right\|_{h}} \geq \beta .
$$

Moreover, $\beta=\mathcal{O}(\delta)$, if $\delta \ll 1$, and $\beta=\mathcal{O}\left(\frac{\mu}{r_{\min }}\right)$, if $\frac{r_{\min }}{\mu} \gg 1$.

Proof. Using the definition of $B_{\delta, \mathbf{R}_{\gamma}}$ and the notations

$$
x=\left\{\delta \sum_{T \in \mathcal{T}_{h}} \frac{h^{2}}{\mu}\left\|\nabla p_{h}\right\|_{T}^{2}\right\}^{1 / 2}, \quad y=\left\{\delta \sum_{E \in \mathcal{G}_{h}} \frac{h}{\mu}\left\|\llbracket p_{h} \rrbracket\right\|_{E}^{2}\right\}^{1 / 2},
$$

we have

$$
\begin{aligned}
B_{\delta, \mathbf{R}_{\gamma}}\left(\left(\mathbf{u}_{h}, p_{h}\right),\left(\mathbf{u}_{h}, p_{h}\right)\right) \geq & \left|\mu^{1 / 2} \mathbf{u}_{h}\right|_{1, \Omega}^{2}+\left\|r_{\min }^{1 / 2} \mathbf{u}_{h}\right\|_{0, \gamma}^{2}+x^{2}+y^{2}+\delta \sum_{T \in \mathcal{T}_{h}} h^{2}\left(-\Delta \mathbf{u}_{h}, \nabla p_{h}\right)_{T} \\
& +\delta \sum_{E \in \mathcal{G}_{h}} \frac{h}{\mu}\left(\llbracket \mu \nabla \mathbf{u}_{h} \cdot \mathbf{n} \rrbracket+\mathbf{R}_{\gamma} \mathbf{u}_{h},-\llbracket p_{h} \mathbf{n} \rrbracket\right)_{E},
\end{aligned}
$$

for all $\left(\mathbf{u}_{h}, p_{h}\right) \in \mathbf{V}_{h}^{k} \times M_{h}^{k}$. Using the inverse estimates (2.12) and (2.13), it follows that

$$
\begin{aligned}
B_{\delta, \mathbf{R}_{\gamma}}\left(\left(\mathbf{u}_{h}, p_{h}\right),\left(\mathbf{u}_{h}, p_{h}\right)\right) \geq & \left|\mu^{1 / 2} \mathbf{u}_{h}\right|_{1, \Omega}^{2}+\left\|r_{\min }^{1 / 2} \mathbf{u}_{h}\right\|_{0, \gamma}^{2}+x^{2}+y^{2}-\delta^{1 / 2} c_{5}\left(\mu\left|\mathbf{u}_{h}\right|_{1, \Omega}^{2}\right)^{1 / 2} x \\
& -\delta^{1 / 2} c_{6}\left(\mu\left|\mathbf{u}_{h}\right|_{1, \Omega}^{2}\right)^{1 / 2} y-\left(\delta \frac{r_{\max }^{2} h}{r_{\min } \mu}\right)^{1 / 2}\left\|r_{\min }^{1 / 2} \mathbf{u}_{h}\right\|_{0, \gamma} y \\
\geq & {\left[1-\delta\left(\frac{c_{5}^{2}}{2}+c_{6}^{2}\right)\right]\left|\mu^{1 / 2} \mathbf{u}_{h}\right|_{1, \Omega}^{2}+\left(1-\delta \frac{r_{\max }^{2} h}{r_{\min } \mu}\right)\left\|r_{\min }^{1 / 2} \mathbf{u}_{h}\right\|_{0, \gamma}^{2} } \\
& +\frac{1}{2} x^{2}+\frac{1}{2} y^{2} .
\end{aligned}
$$

Finally, using (2.19), we get

$$
B_{\delta, \mathbf{R}_{\gamma}}\left(\left(\mathbf{u}_{h}, p_{h}\right),\left(\mathbf{u}_{h}, p_{h}\right)\right) \geq \frac{1}{2}\left\{\left|\mu^{1 / 2} \mathbf{u}_{h}\right|_{1, \Omega}^{2}+\left\|r_{\min }^{1 / 2} \mathbf{u}_{h}\right\|_{0, \gamma}^{2}+x^{2}+y^{2}\right\},
$$

for all $\left(\mathbf{u}_{h}, p_{h}\right) \in \mathbf{V}_{h}^{k} \times M_{h}^{k}$, which provides the coercivity of the stabilized bi-linear form $B_{\delta, \mathbf{R}_{\gamma}}$. 
Let now $\left(\mathbf{u}_{h}, p_{h}\right) \in \mathbf{V}_{h}^{k} \times M_{h}^{k}$ fixed. There exists a $\mathbf{v} \in \mathbf{V}$ and a constant $c_{\Omega}$, which only depends on $\Omega$, such that div $\mathbf{v}=-p_{h}$ and $\|\mathbf{v}\|_{1, \Omega} \leq c_{\Omega}\left\|p_{h}\right\|_{0, \Omega}$, see for instance [19]. Let $\mathbf{v}_{h}=S Z_{h}^{k} \mathbf{v}$. Using the $H^{1}$-stability of $S Z_{h}^{k}$ (see [24] or [13], p. 71) we have

$$
\left\|\mathbf{v}_{h}\right\|_{1, \Omega} \leq c_{S Z}\|\mathbf{v}\|_{1, \Omega} \leq c_{\Omega}^{\prime}\left\|p_{h}\right\|_{0, \Omega} .
$$

Using partial integration element-wise and the continuity of $p_{h}$ in $\Omega_{1}$ and $\Omega_{2}$, one obtains

$$
\begin{aligned}
B_{\delta, \mathbf{R}_{\gamma}}\left(\left(\mathbf{u}_{h}, p_{h}\right),\left(\mathbf{v}_{h}, 0\right)\right)= & \left(\mu \nabla \mathbf{u}_{h}, \nabla \mathbf{v}_{h}\right)_{\Omega}+\left(\mathbf{R}_{\gamma} \mathbf{u}_{h}, \mathbf{v}_{h}\right)_{\gamma}-\left(p_{h}, \operatorname{div} \mathbf{v}\right)_{\Omega} \\
& -\sum_{T \in \mathcal{T}_{h}}\left(\nabla p_{h}, \mathbf{v}-\mathbf{v}_{h}\right)_{T}+\sum_{E \in \mathcal{G}_{h}}\left(\llbracket p_{h} \mathbf{n} \rrbracket, \mathbf{v}-\mathbf{v}_{h}\right)_{E} .
\end{aligned}
$$

Therefore, using Cauchy-Schwarz and approximation (2.6)-(2.7), one obtains

$$
\begin{aligned}
B_{\delta, \mathbf{R}_{\gamma}}\left(\left(\mathbf{u}_{h}, p_{h}\right),\left(\mathbf{v}_{h}, 0\right)\right) \geq & \left\|p_{h}\right\|_{0, \Omega}^{2}-\left|\mu^{1 / 2} \mathbf{u}_{h}\right|_{1, \Omega}\left|\mu^{1 / 2} \mathbf{v}_{h}\right|_{1, \Omega}-\frac{r_{\max }}{r_{\min }^{1 / 2}}\left\|r_{\min }^{1 / 2} \mathbf{u}_{h}\right\|_{0, \gamma}\left\|\mathbf{v}_{h}\right\|_{0, \gamma} \\
& -\left[c_{0}\left(\sum_{T \in \mathcal{T}_{h}} h^{2}\left\|\nabla p_{h}\right\|_{0, T}^{2}\right)^{1 / 2}+C_{0}\left(\sum_{E \in \mathcal{G}_{h}} h\left\|\llbracket p_{h} \rrbracket\right\|_{0, E}^{2}\right)^{1 / 2}\right]\|\mathbf{v}\|_{1, \Omega} .
\end{aligned}
$$

It then follows that

$$
\begin{aligned}
B_{\delta, \mathbf{R}_{\gamma}}\left(\left(\mathbf{u}_{h}, p_{h}\right),\left(\mathbf{v}_{h}, 0\right)\right) \geq & \left\|p_{h}\right\|_{0, \Omega}^{2}-c_{\Omega}^{\prime} \mu^{1 / 2}\left|\mu^{1 / 2} \mathbf{u}_{h}\right|_{1, \Omega}\left\|p_{h}\right\|_{0, \Omega}-c_{\Omega}^{\prime} c_{\gamma} \frac{r_{\max }}{r_{\min }^{1 / 2}}\left\|r_{\min }^{1 / 2} \mathbf{u}_{h}\right\|_{0, \gamma}\left\|p_{h}\right\|_{0, \Omega} \\
& -\left(\frac{\mu}{\delta}\right)^{1 / 2} c_{0} c_{\Omega} x\left\|p_{h}\right\|_{0, \Omega}-\left(\frac{\mu}{\delta}\right)^{1 / 2} C_{0} c_{\Omega} y\left\|p_{h}\right\|_{0, \Omega} \\
\geq & \left\|p_{h}\right\|_{0, \Omega}^{2}-c_{\delta} \mu^{1 / 2}\left(\left|\mu^{1 / 2} \mathbf{u}_{h}\right|_{1, \Omega}+\left\|r_{\min }^{1 / 2} \mathbf{u}_{h}\right\|_{0, \gamma}+x+y\right)\left\|p_{h}\right\|_{0, \Omega} \\
\geq & \frac{1}{2}\left\|p_{h}\right\|_{0, \Omega}^{2}-2 c_{\delta}^{2} \mu\left(\left|\mu^{1 / 2} \mathbf{u}_{h}\right|_{1, \Omega}^{2}+\left\|r_{\min }^{1 / 2} \mathbf{u}_{h}\right\|_{0, \gamma}^{2}+x^{2}+y^{2}\right)
\end{aligned}
$$

where

$$
c_{\delta}=\max \left\{c_{\Omega}^{\prime}, c_{\Omega}^{\prime} c_{\gamma} \frac{r_{\max }}{\mu^{1 / 2} r_{\min }^{1 / 2}}, \delta^{-1 / 2} c_{0} c_{\Omega}, \delta^{-1 / 2} C_{0} c_{\Omega}\right\} .
$$

Multiplying (2.21) by $(1-\rho)$ and $(2.22)$ by $\rho$, and adding the results, one obtains

$$
\begin{array}{r}
B_{\delta, \mathbf{R}_{\gamma}}\left(\left(\mathbf{u}_{h}, p_{h}\right),\left((1-\rho) \mathbf{u}_{h}+\rho \mathbf{v}_{h},(1-\rho) p_{h}\right)\right)=(1-\rho) B_{\delta, \mathbf{R}_{\gamma}}\left(\left(\mathbf{u}_{h}, p_{h}\right),\left(\mathbf{u}_{h}, p_{h}\right)\right)+\rho B_{\delta, \mathbf{R}_{\gamma}}\left(\left(\mathbf{u}_{h}, p_{h}\right),\left(\mathbf{v}_{h}, 0\right)\right) \\
\geq\left(\frac{1-\rho}{2}-2 \rho c_{\delta}^{2} \mu\right)\left(\mu\left|\mathbf{u}_{h}\right|_{1}^{2}+\left\|\left(r_{\min }\right)^{1 / 2} \mathbf{u}\right\|_{0, \gamma}^{2}+x^{2}+y^{2}\right)+\frac{\rho \mu}{2}\left\|\mu^{-1 / 2} p_{h}\right\|_{0, \Omega}^{2} .
\end{array}
$$

So that, if we take

we then obtain that

$$
0<\rho=\frac{1}{1+4 c_{\delta}^{2} \mu+\mu}<1
$$

$$
B_{\delta, \mathbf{R}_{\gamma}}\left(\left(\mathbf{u}_{h}, p_{h}\right),\left((1-\rho) \mathbf{u}_{h}+\rho \mathbf{v}_{h},(1-\rho) p_{h}\right)\right) \geq \frac{\mu}{2+8 c_{\delta}^{2} \mu+2 \mu}\left\|\left(\mathbf{u}_{h}, p_{h}\right)\right\|_{h}^{2} .
$$


Moreover,

$$
\begin{aligned}
\left\|\left((1-\rho) \mathbf{u}_{h}+\rho \mathbf{v}_{h},(1-\rho) p_{h}\right)\right\|_{h} & \leq(1-\rho)\left\|\left(\mathbf{u}_{h}, p_{h}\right)\right\|_{h}+\rho\left\|\left(\mathbf{v}_{h}, 0\right)\right\|_{h} \\
& \leq(1-\rho)\left\|\left(\mathbf{u}_{h}, p_{h}\right)\right\|_{h}+\rho\left\{\mu\left|\mathbf{v}_{h}\right|_{1, \Omega}^{2}+\left\|r_{\min }^{1 / 2} \mathbf{v}_{h}\right\|_{0, \gamma}^{2}\right\}^{1 / 2} \\
& \leq(1-\rho)\left\|\left(\mathbf{u}_{h}, p_{h}\right)\right\|_{h}+\rho \sqrt{2} c_{\delta} \mu\left\|\mu^{-1 / 2} p_{h}\right\|_{0, \Omega} \\
& \leq \mu \frac{1+\sqrt{2} c_{\delta}+4 c_{\delta}^{2}}{1+4 c_{\delta}^{2} \mu+\mu}\left\|\left(\mathbf{u}_{h}, p_{h}\right)\right\|_{h} .
\end{aligned}
$$

Combining (2.23) and (2.24), one obtains the desired inf-sup estimation with

$$
\beta=\frac{1}{2+2 \sqrt{2} c_{\delta}+8 c_{\delta}^{2}} .
$$

The asymptotic behavior of $\beta$ follows from this equation and the definition of $c_{\delta}$, so the proof is complete.

Remark 2.4. The proof of the above theorem points out the purpose of the stabilizing term, giving $L^{2}$-stability of the pressure jumps through the interface (as shown in (2.21)). Indeed, it allows to keep under control the last term of (2.22) and, as a result, to obtain an inf-sup constant $\beta$ independent of $h$. To the best of our knowledge, there is no clear-cut evidence that such a (uniform) stability result can be achieved without the addition of an (extra) control on the pressure jump.

As a direct consequence of the previous result, we have the following corollary.

Corollary 2.5. There exists a unique solution to problem (2.14).

\subsubsection{Convergence analysis}

In this paragraph we provide an optimal error estimate under the reduced regularity assumptions (2.5). First, we prove the following approximability result with respect to the mesh-dependent norm $\|\cdot\|_{h}$.

Proposition 2.6. Assume that $(\mathbf{u}, p)$ satisfies (2.5). Then, there exists a positive constant $c$, independent of $h$ and the physical parameters, such that:

$$
\left\|\left(\mathbf{u}-I_{h}^{k} \mathbf{u}, p-J_{h}^{k} p\right)\right\|_{h} \leq c h^{k}\left[\left(\mu^{1 / 2}+r_{\min }^{1 / 2} h^{1 / 2}\right) \mathcal{N}_{k+1}(\mathbf{u})+\left(1+\delta^{1 / 2}\right) \mu^{-1 / 2} \mathcal{N}_{k}(p)\right],
$$

with the notations

$$
\mathcal{N}_{k+1}(\mathbf{u})=\sum_{i=1,2}\|\mathbf{u}\|_{k+1, \Omega_{i}}, \quad \mathcal{N}_{k}(p)=\sum_{i=1,2}\|p\|_{k, \Omega_{i}} .
$$

Proof. Using the definition (2.18) and the approximation properties of $I_{h}^{k}$ and $J_{h}^{k}(2.8)$ and (2.11), we have

$$
\begin{aligned}
\left\|\left(\mathbf{u}-I_{h}^{k} \mathbf{u}, p-J_{h}^{k} p\right)\right\|_{h}= & \left\{\left|\mu^{1 / 2}\left(\mathbf{u}-I_{h}^{k} \mathbf{u}\right)\right|_{1, \Omega}^{2}+\left\|\mu^{-1 / 2}\left(p-J_{h}^{k} p\right)\right\|_{0, \Omega}^{2}+\left\|r_{\min }^{1 / 2}\left(\mathbf{u}-I_{h}^{k} \mathbf{u}_{h}\right)\right\|_{0, \gamma}^{2}\right. \\
& \left.+\delta \frac{h^{2}}{\mu} \sum_{i=1,2}\left\|\nabla\left(p-J_{h}^{k} p\right)\right\|_{0, \Omega_{i}}^{2}+\delta \frac{h}{\mu}\left\|\llbracket p-J_{h}^{k} p \rrbracket\right\|_{0, \gamma}^{2}\right\}^{1 / 2} \\
\leq & \left(c_{1} \mu^{1 / 2} h^{k}+c_{2} r_{\min }^{1 / 2} h^{k+1 / 2}\right) \mathcal{N}_{k+1}(\mathbf{u}) \\
& +\left(c_{3} \mu^{-1 / 2} h^{k}+\delta^{1 / 2} \mu^{-1 / 2}\left(c_{3}+c_{4}\right) h^{k}\right) \mathcal{N}_{k}(p) \\
\leq & c h^{k}\left[\left(\mu^{1 / 2}+r_{\min }^{1 / 2} h^{1 / 2}\right) \mathcal{N}_{k+1}(\mathbf{u})+\left(\mu^{-1 / 2}+\delta^{1 / 2} \mu^{-1 / 2}\right) \mathcal{N}_{k}(p)\right],
\end{aligned}
$$

which completes the proof. 
The main result of this paragraph is stated in the next theorem.

Theorem 2.7. Let $(\mathbf{u}, p) \in \mathbf{V} \times \tilde{M}$ be the solution of problem (2.3), and $\left(\mathbf{u}_{h}, p_{h}\right) \in \mathbf{V}_{h} \times M_{h}^{k}$ the solution of problem (2.14). Assume that the hypotheses of Theorem 2.3 hold. Then, there exists a positive constant $c$, independent of $h$, depending on the inf-sup constant $\beta$, such that

$$
\begin{aligned}
\left\|\left(\mathbf{u}-\mathbf{u}_{h}, p-p_{h}\right)\right\|_{h} \leq & c(\beta) h^{k}\left\{\left(\mu^{1 / 2}+\delta^{1 / 2} \mu^{1 / 2}+\delta^{-1 / 2} \mu^{1 / 2}\right.\right. \\
& \left.+\frac{r_{\max }}{r_{\min }^{1 / 2}} h^{1 / 2}+\delta^{1 / 2} \mu^{-1 / 2} r_{\max } h\right) \sum_{i=1,2}\|\mathbf{u}\|_{k+1, \Omega_{i}} \\
& \left.+\left(\mu^{-1 / 2}+\delta^{1 / 2} \mu^{-1 / 2}\right) \sum_{i=1,2}\|p\|_{k, \Omega_{i}}\right\}
\end{aligned}
$$

Proof. Take the interpolants $\mathbf{v}_{h}=I_{h}^{k} \mathbf{u}$ and $q_{h}=J_{h}^{k} p$. Decompose the error into the interpolation and approximation errors,

$$
\left\|\left(\mathbf{u}-\mathbf{u}_{h}, p-p_{h}\right)\right\|_{h} \leq\left\|\left(\mathbf{u}-\mathbf{v}_{h}, p-q_{h}\right)\right\|_{h}+\left\|\left(\mathbf{u}_{h}-\mathbf{v}_{h}, p_{h}-q_{h}\right)\right\|_{h} .
$$

The first term can be bounded using Proposition 2.6, so we only need to estimate $\left\|\left(\mathbf{u}_{h}-\mathbf{v}_{h}, p_{h}-q_{h}\right)\right\|_{h}$. Using the inf-sup condition (Thm. 2.3) and the Galerkin orthogonality (2.16), we have

$$
\begin{aligned}
\left\|\left(\mathbf{u}_{h}-\mathbf{v}_{h}, p_{h}-q_{h}\right)\right\|_{h} & \leq \frac{1}{\beta} \sup _{\left(\mathbf{w}_{h}, r_{h}\right) \in \mathbf{V}_{h}^{k} \times M_{h}^{k}} \frac{B_{\delta, \mathbf{R}_{\gamma}}\left(\left(\mathbf{u}_{h}-\mathbf{v}_{h}, p_{h}-q_{h}\right),\left(\mathbf{w}_{h}, r_{h}\right)\right)}{\left\|\left(\mathbf{w}_{h}, r_{h}\right)\right\|_{h}} \\
& \leq \frac{1}{\beta} \sup _{\left(\mathbf{w}_{h}, r_{h}\right) \in \mathbf{V}_{h}^{k} \times M_{h}^{k}} \frac{B_{\delta, \mathbf{R}_{\gamma}}\left(\left(\mathbf{u}-\mathbf{v}_{h}, p-q_{h}\right),\left(\mathbf{w}_{h}, r_{h}\right)\right)}{\left\|\left(\mathbf{w}_{h}, r_{h}\right)\right\|_{h}} .
\end{aligned}
$$

To estimate the right-hand side of the above inequality, we take $\left(\mathbf{w}_{h}, r_{h}\right) \in \mathbf{V}_{h}^{k} \times M_{h}^{k}$ arbitrarily, and we bound separately each term of $B_{\delta, \mathbf{R}_{\gamma}}\left(\left(\mathbf{u}-\mathbf{v}_{h}, p-q_{h}\right),\left(\mathbf{w}_{h}, r_{h}\right)\right)$, using approximation estimates (2.8) and (2.11). For the viscous term, we have

$$
\left(\mu \nabla\left(\mathbf{u}-\mathbf{v}_{h}\right), \nabla \mathbf{w}_{h}\right)_{\Omega} \leq c_{1} h^{k} \mu^{1 / 2} \mathcal{N}_{k+1}(\mathbf{u})\left\|\left(\mathbf{w}_{h}, r_{h}\right)\right\|_{h} .
$$

The resistive term is treated as follows

$$
\begin{aligned}
\left(\mathbf{R}_{\gamma}\left(\mathbf{u}-\mathbf{v}_{h}\right), \mathbf{w}_{h}\right)_{\gamma} & \leq\left\|\mathbf{R}_{\gamma}{ }^{1 / 2}\left(\mathbf{u}-\mathbf{v}_{h}\right)\right\|_{0, \gamma}\left\|\mathbf{R}_{\gamma}{ }^{1 / 2} \mathbf{w}_{h}\right\|_{0, \gamma} \\
& \leq c_{2} \frac{r_{\max }}{r_{\min }^{1 / 2}} h^{k+1 / 2} \mathcal{N}_{k+1}(\mathbf{u})\left\|\left(\mathbf{w}_{h}, r_{h}\right)\right\|_{h} .
\end{aligned}
$$

For the pressure term, we have

$$
-\left(p-q_{h}, \operatorname{div} \mathbf{w}_{h}\right)_{\Omega} \leq c_{3} \mu^{-1 / 2} h^{k} \mathcal{N}_{k}(p) \sqrt{n}\left\|\left(\mathbf{w}_{h}, r_{h}\right)\right\|_{h} .
$$

By integration by parts, we obtain

$$
\begin{aligned}
\left(r_{h}, \operatorname{div}\left(\mathbf{u}-\mathbf{v}_{h}\right)\right)_{\Omega} & =-\sum_{T \in \mathcal{T}_{h}}\left(\nabla r_{h}, \mathbf{u}-\mathbf{v}_{h}\right)_{T}+\sum_{E \in \mathcal{G}_{h}}\left(\mathbf{u}-\mathbf{v}_{h}, \llbracket r_{h} \mathbf{n} \rrbracket\right)_{E} \\
& \leq\left(c_{1}+c_{2}\right) h^{k} \mathcal{N}_{k+1}(\mathbf{u}) \delta^{-1 / 2} \mu^{1 / 2}\left\|\left(\mathbf{w}_{h}, r_{h}\right)\right\|_{h} .
\end{aligned}
$$


For the stabilization terms, we have

$$
\delta \sum_{T \in \mathcal{T}_{h}} \frac{h^{2}}{\mu}\left(-\mu \Delta\left(\mathbf{u}-\mathbf{v}_{h}\right)+\nabla\left(p-q_{h}\right), \nabla r_{h}\right)_{T} \leq h^{k}\left(c_{1} \delta^{1 / 2} \mu^{1 / 2} \mathcal{N}_{k+1}(\mathbf{u})+c_{3} \delta^{1 / 2} \mu^{-1 / 2} \mathcal{N}_{k}(p)\right)\left\|\left(\mathbf{w}_{h}, r_{h}\right)\right\|_{h}
$$

On the other hand, using the fact that $\nabla \mathbf{u} \in H^{k}\left(\Omega_{i}\right)^{n \times n}, i=1,2$ and since

$$
\left\|\llbracket \nabla\left(\mathbf{u}-\mathbf{v}_{h}\right) \cdot \mathbf{n} \rrbracket\right\|_{0, E} \leq\left\|\llbracket \nabla\left(\mathbf{u}-\mathbf{v}_{h}\right) \rrbracket\right\|_{0, E} \leq c_{2} h^{k-1 / 2} \sum_{i=1,2}\|\nabla \mathbf{u}\|_{k, T_{i}}, \quad E=T_{1} \cap T_{2},
$$

we obtain

$$
\begin{aligned}
\delta \sum_{E \in \mathcal{G}_{h}} \frac{h}{\mu}\left(\llbracket \mu \nabla\left(\mathbf{u}-\mathbf{v}_{h}\right) \cdot \mathbf{n} \rrbracket,-\llbracket r_{h} \mathbf{n} \rrbracket\right)_{E} & \leq c_{2} h^{k} \delta^{1 / 2} \mu^{1 / 2} \mathcal{N}_{k+1}(\mathbf{u})\left\|\left(\mathbf{w}_{h}, r_{h}\right)\right\|_{h}, \\
\delta \sum_{E \in \mathcal{G}_{h}} \frac{h}{\mu}\left(\mathbf{R}_{\gamma}\left(\mathbf{u}-\mathbf{v}_{h}\right),-\llbracket r_{h} \mathbf{n} \rrbracket\right)_{E} & \leq c_{2} h^{k+1} \delta^{1 / 2} \mu^{-1 / 2} r_{\max } \mathcal{N}_{k+1}(\mathbf{u})\left\|\left(\mathbf{w}_{h}, r_{h}\right)\right\|_{h}, \\
\delta \sum_{E \in \mathcal{G}_{h}} \frac{h}{\mu}\left(\llbracket-\left(p-q_{h}\right) \mathbf{n} \rrbracket,-\llbracket r_{h} \mathbf{n} \rrbracket\right)_{E} & \leq c_{4} h^{k} \delta^{1 / 2} \mu^{-1 / 2} \mathcal{N}_{k}(p)\left\|\left(\mathbf{w}_{h}, r_{h}\right)\right\|_{h} .
\end{aligned}
$$

We conclude the proof by summing up all the contributions.

\section{NUMERICAL RESULTS}

This section is devoted to numerical results. In Section 3.1, we assess the theoretical convergence rates stated in Section 2.3 with an analytical test-case. In Section 3.2, we consider a quasi-Poiseuille flow through a porous surface using different discretization spaces. The purpose is to stress the relevance of using discontinuous pressures across the interface and to show that the proposed stabilized finite element provides solutions similar to those obtained with more expensive finite elements. Finally, in Section 3.3, we show the results of simulations with a realistic aneurysm geometry, both with rigid and elastic walls.

\subsection{Assessment of the convergence rate}

We build an analytical solution of the problem in order to assess the convergence rate proved above. Let $\Omega=(0,2) \times(0,1)$ be the fluid domain divided in two subdomains $\Omega_{1}=(0, L) \times(0,1)$ and $\Omega_{2}=(L, 2) \times(0,1)$, by the interface $\gamma=\{L\} \times(0,1)$, where $L=1$. Assume that the viscosity $\mu=0.04$ and the resistivity is the scalar matrix $\mathbf{R}_{\gamma}=100 \mathbf{I}$. We compute $\mathbf{f}$ and the non-homogeneous boundary conditions such that the following functions are solution to problem (2.1):

$$
\mathbf{u}^{1}=\left[\begin{array}{c}
-19.98 x+10 x^{2} \\
-40.04+19.98 y+40 x^{2}-20 x y
\end{array}\right], \mathbf{u}^{2}=\left[\begin{array}{c}
25-69.98 x+35 x^{2} \\
9.96+69.98 y-10 x^{2}-70 x y
\end{array}\right]
$$

and

$$
p^{1}=800 y^{2}, p^{2}=998+800 y^{2}
$$

where $\left(\mathbf{u}^{i}, p^{i}\right)$ denotes the solution in $\Omega_{i}$. This solution is depicted in Figure 4. The functions $\left(\mathbf{u}^{i}, p^{i}\right), i=1,2$, were chosen as polynomials of degree 2 in $(x, y)$, that satisfy the model problem (2.1) and enjoy a large pressure jump at the interface and a moderate jump of the normal derivatives of the velocity. This corresponds to the typical physical conditions we consider in this paper.

Various unstructured triangulations with decreasing mesh parameters $h \in\{1 / 10 ; 1 / 20 ; 1 / 40 ; 1 / 80\}$ have been considered and the results are reported in Figure 5. 

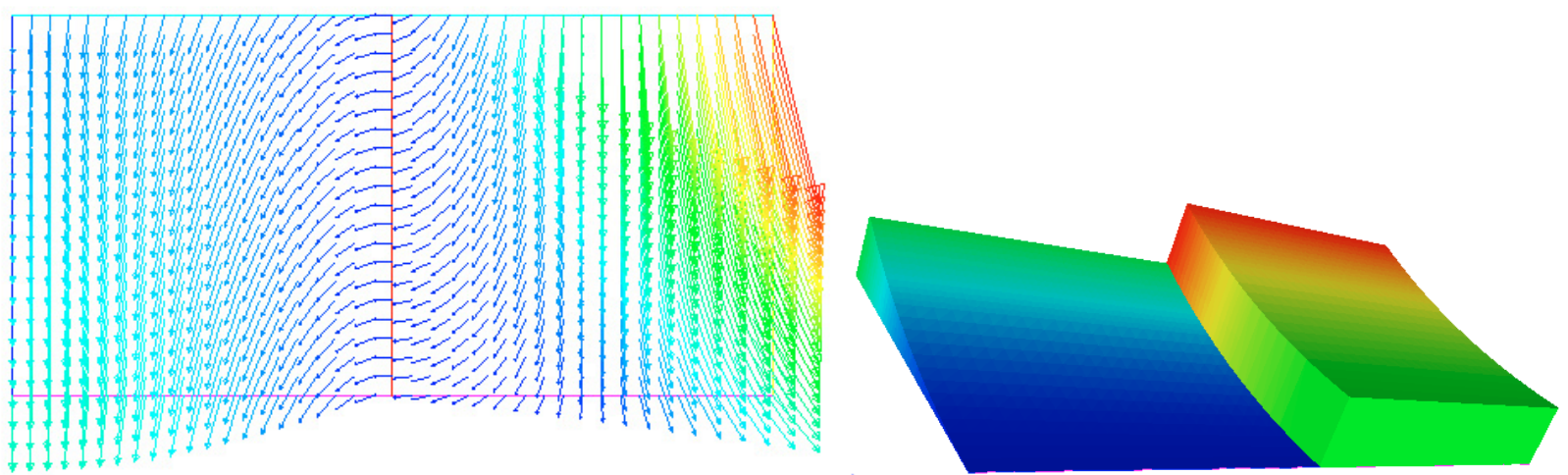

FigURE 4. Velocity (left) and pressure (right) with a resistive stent (red vertical line, $r_{\gamma}=100$ ) corresponding to the Section 3.1 test-case. Discretization: $P_{1} / P_{1}$ with a fissure. Velocity scale: from 9.63 up to 103.1. Pressure scale: from 0 up to 1800. The mesh presented in this figure is quite coarse for clarity.
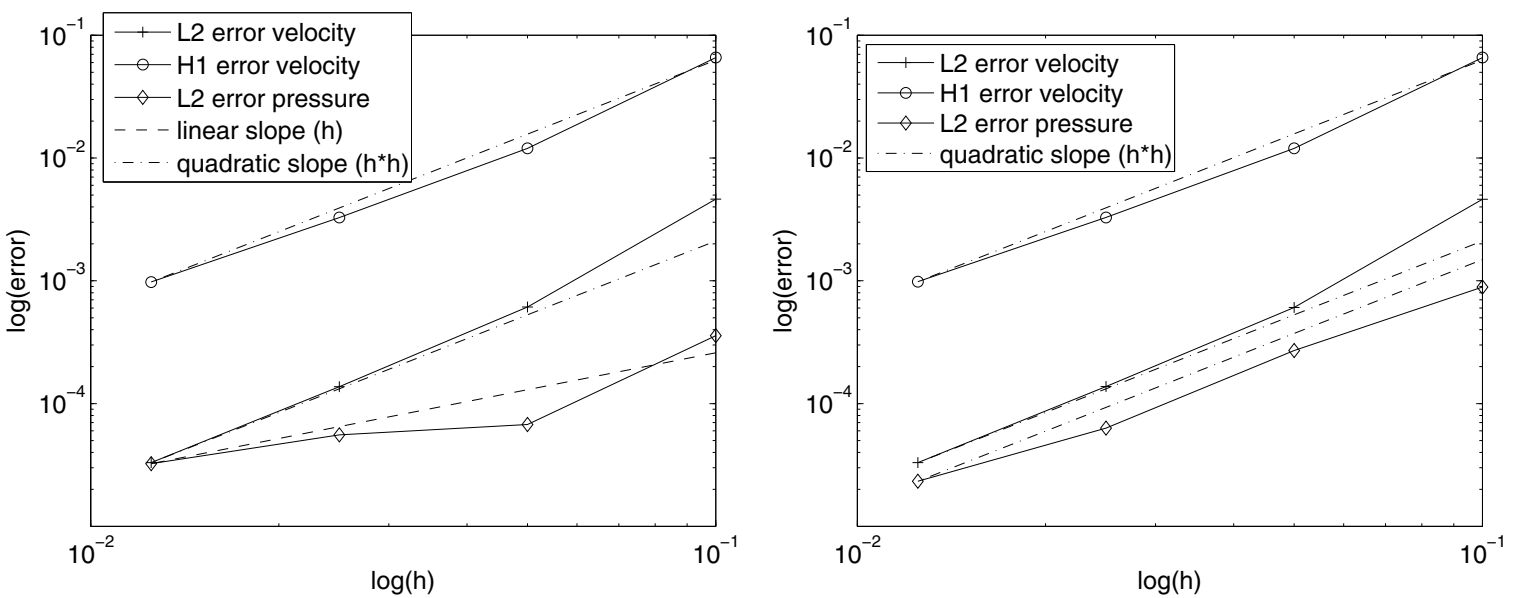

Figure 5. Test-case of Section 3.1. Relative error as a function of the discretization step $h$, in log scales. Left: classical residual based stabilization. Right: stabilization of Section 2.3.

According to the results proved in the previous section, the convergence of the formulation including a stabilization of the pressure jump over $\gamma$ is expected to be optimal. This property essentially comes from the fact that the inf-sup constant in Theorem 2.3 is independent of $h$. On the other hand, when the additional term stabilizing the pressure jump is not considered, we were not able to obtain an inf-sup constant independent of $h$. It is therefore expected that the convergence rate without pressure jump stabilization is lower than with the formulation proposed in this work. Figure 5 confirms this expectation: a comparison with the "classical" residual based stabilization's (Fig. 5, left) shows that the proposed stabilization method performs similarly as far as the velocity is concerned, but enjoys a better convergence rate for the pressure.

Note that we do not explain the super-convergence observed for the velocity gradient and the pressure. The only purpose of this simple test case was just to illustrate the fact that the proposed formulation may improve the pressure convergence rate. 


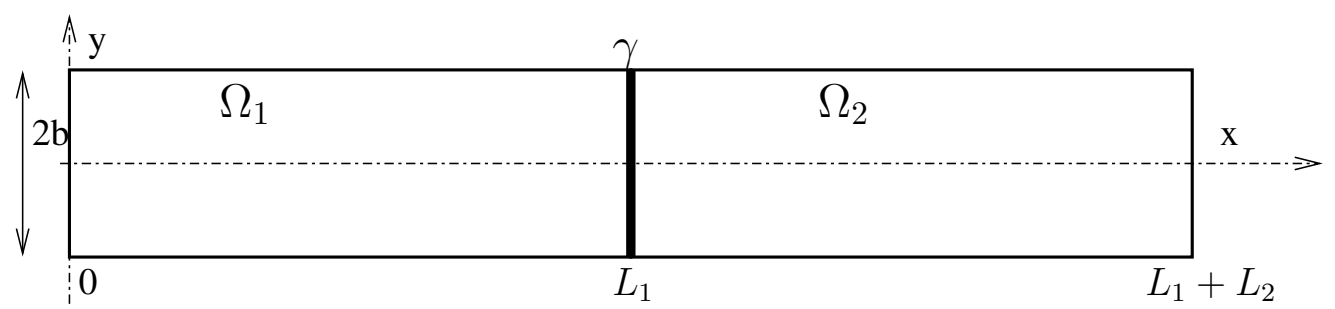

Figure 6. Quasi-Poiseuille test-case: the subdomains $\Omega_{1}$ and $\Omega_{2}$ are separated by the stent considered as a straight line $\gamma$.

\subsection{Quasi-Poiseuille flow}

\subsubsection{Description of the problem}

We consider a $2 \mathrm{D}$ stationary flow in a straight $2 \mathrm{D}$ tube of length $L=L_{1}+L_{2}, L_{1}>0$ and $L_{2}>0$, of width $2 b>0$, with a stent $\gamma$ located inside the tube, see Figure 6 . We decomposed the domain $\Omega=(0, L) \times(-b, b)$ into two subdomains $\Omega_{1}=\left(0, L_{1}\right) \times(-b, b)$ and $\Omega_{2}=\left(L_{1}, L\right) \times(-b, b)$. The stent is modeled by a resistivity $\mathbf{R}_{\gamma}=r_{\gamma} \mathbf{I}$ at the interface $\gamma=\left\{L_{1}\right\} \times(-b, b)$.

We impose a no-slip boundary condition on the wall of the tube, $\mathbf{u}(x, b)=\mathbf{u}(x,-b)=0, x \in[0, L]$ and the following natural boundary conditions at the inlet and the outlet:

$$
\mathbf{T}(\mathbf{u}, p)(0, y) \cdot \mathbf{n}=-P_{0} \mathbf{n}, \quad \mathbf{T}(\mathbf{u}, p)(L, y) \cdot \mathbf{n}=-P_{L} \mathbf{n}, \quad y \in[-b, b] .
$$

Remark 3.1. In absence of stent (namely if $r_{\gamma}=0$ ), the solution to this problem is the standard Poiseuille flow with a linear decreasing pressure and a parabolic velocity profile. Of course, this does not hold true if $r_{\gamma}>0$, since, in particular, if the pressure was constant over a tube section, then the fourth equation in (2.1) would yield a flat velocity profile on the interface $\gamma$ (which is incompatible with the parabolic profile of Poiseuille flows). Nevertheless, as a first approximation, if we assume that a Poiseuille flow is established in $\Omega_{1}$ and $\Omega_{2}$ then, we readily obtain

$$
\bar{u}=-\frac{b^{2}}{3 \mu L_{1}}\left(P_{1}-P_{0}\right)=-\frac{1}{r_{\gamma}}\left(P_{2}-P_{1}\right)=-\frac{b^{2}}{3 \mu L_{2}}\left(P_{L}-P_{2}\right),
$$

with $\bar{u}=\frac{1}{2 b} \int_{-b}^{b} u(y) \mathrm{d} y$, and $P_{1}=P\left(L_{1}^{-}\right), P_{2}=P\left(L_{1}^{+}\right)$. Thus introducing $R_{i}=\frac{3 \mu L_{i}}{b^{2}}, i=1,2$, we have

$$
P_{0}-P_{L}=\left(R_{1}+r_{\gamma}+R_{2}\right) \bar{u}
$$

In particular this relation can be used to evaluate experimentally the parameter $r_{\gamma}$ of real stents. Our computations have been performed with realistic values obtained in this way.

The following parameters are considered for the numerical simulations. The dimensions of the tube are $L_{1}=$ $L_{2}=4 \mathrm{~cm}, 2 b=0.4 \mathrm{~cm}$, the viscosity is $\mu=0.04 \mathrm{~g} \cdot \mathrm{cm}^{-1} \cdot \mathrm{s}^{-1}$ ) and the stent resistivity is $r_{\gamma}=100 \mathrm{~g} \cdot \mathrm{cm}^{-2} \cdot \mathrm{s}^{-1}$ ). We impose a constant pressure drop $\Delta P=1000 \mathrm{~g} \cdot \mathrm{cm}^{-1} \cdot \mathrm{s}^{-2}$ ) in a transient simulation, and wait for the stationary state to be reached.

According to Remark 3.1, an approximation of the solution is obtained by assuming a Poiseuille flow before and after the stent. The corresponding mean flow and the pressure jump at the interface are readily given by:

$$
\phi^{Q P}=\int_{-b}^{b} u(y) \mathrm{d} y \approx 3.226 \mathrm{~cm}^{3} \cdot \mathrm{s}^{-1}, \quad \Delta P_{\gamma}^{Q P}=P_{1}-P_{2} \approx 806.5 \mathrm{~g} \cdot \mathrm{cm}^{-1} \cdot \mathrm{s}^{-2}
$$

Since no analytical solution is available for this test-case, we compare the mean values of the numerical results with this approximated solution. 
TABLE 1. Effect of the continuity of the pressure at the interface over the $h$ convergence, using different discretization spaces.

\begin{tabular}{||c||c|c|c||c|c|c||}
\hline \multicolumn{1}{||c||}{} & \multicolumn{3}{c||}{ No fissure } & \multicolumn{3}{c||}{ Fissure } \\
\hline$\left.p_{h}\right|_{\gamma}$ & $C^{o}$ & $C^{O}$ & Disc & Disc & Disc & Disc \\
\hline Spaces & $Q_{1} / Q_{1}$ & $Q_{2} / Q_{1}$ & $Q_{2} / P_{1}$ & $Q_{1} / Q_{1}$ & $Q_{2} / Q_{1}$ & $Q_{2} / P_{1}$ \\
\hline Convergence & no CV & slow & $O K$ & $O K$ & $O K$ & $O K$ \\
\hline
\end{tabular}

The mesh is made of structured quadrangles. It is supposed to be conforming at the porous interface, i.e. the interface is made of edges of the global mesh. The pressure is expected to be discontinuous through the interface. There are basically two options to build an approximation space which contains this discontinuity. Option 1: we consider pressure functions which are discontinuous at each element interfaces. Option 2: we consider pressure functions which are continuous over the whole domain, except on the porous interface where we introduce additional pressure degrees of freedom. In the second option, we will say that we introduce a fissure in the mesh. On the fissure, each geometrical point is associated to two vertices.

In Table 1, we summarize the results obtained with different types of discretization spaces:

- stable finite elements: $Q_{2} / Q_{1}$ or $Q_{2} / P_{1}$ (velocity/pressure);

- stabilized finite element: $Q_{1} / Q_{1}$.

We refer to [19] or [13] for the precise definitions of these spaces. We just recall that the pressure is discontinuous through element interfaces for the $Q_{2} / P_{1}$ pair, whereas it is continuous for the $Q_{2} / Q_{1}$ and the stabilized $Q_{1} / Q_{1}$ pairs. Thus, the choice $Q_{2} / P_{1}$ corresponds to the "option 1" explained above. When $Q_{2} / Q_{1}$ or $Q_{1} / Q_{1}$ are considered, it is necessary to introduce a fissure if we want the discontinuous pressure to be included in the approximation space ("option 2").

In the first column of Table 1, the mesh has no fissure on the interface. Thus, the pressure is discontinuous on the interface only with the $Q_{2} / P_{1}$ pair of finite element. In the second column, the mesh contains a fissure at the interface. In this case, the pressure is always discontinuous at the interface and, as far as the $Q_{1} / Q_{1}$ pair is concerned, the stabilization includes the terms introduced in Section 2.3. The qualitative convergence behavior with respect to the mesh refinement is also provided in these different cases.

In Figure 7, we show the velocity around the interface. The velocity profile is almost parabolic in the tube, except near the resistive interface, where it tends to be flat (see Rem. 3.2). In Figure 8, left, the pressure profile along the axis $y=0$ of the tube is reported. The pressure is almost linearly varying in the subdomains, with a strong pressure drop across the interface. In Figure 8, right, one can see the evolution of the outlet flux $\phi=\int_{-b}^{b} u(y) \mathrm{d} y$ as a function of time, until it reaches the stationary state. In both cases, we have drawn (straight lines labelled by "Ref QP") the result predicted by the "quasi-Poiseuille" approximation (see (3.2) and Rem. 3.2).

When the pressure is approximated continuously at the interface, the results are poor: the flux computed in this example is almost twice as large as the correct value, even for a very refined mesh. We note that with the same stabilized $Q_{1} / Q_{1}$ elements, but with a fissure at the interface, a correct solution is computed using the coarsest mesh.

Figure 9 shows the results for the $Q_{2} / Q_{1}$ discretization (left) and the $Q_{2} / P_{1}$ discretization (right). With the $Q_{2} / Q_{1}$ elements, the pressure lies in a continuous space of approximation, and thus one needs either to refine a lot the mesh, or to introduce a fissure in the mesh, to compute the correct solution. With the $Q_{2} / P_{1}$ elements, as the pressure is element-wise discontinuous, the solution is correctly computed with a coarse mesh.

Note that there exists a slight discrepancy between the flux obtained from the "quasi-Poiseuille" flow and the flux computed with the model. This discrepancy is not surprising since the "quasi-Poiseuille" flow is not a solution of the model problem, as clearly shown in Figure 7. Thus, formula (3.1) - which gives the estimation labelled as "Reference Quasi-Poiseuille" - cannot be expected to provide with an exact solution. As shown in 


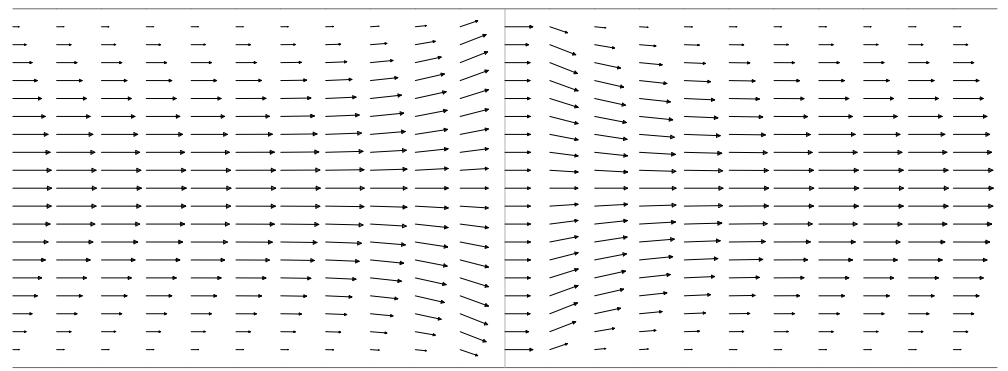

FiguRE 7 . Velocity with a resistive surface $\left(r_{\gamma}=100\right)$ in a $2 \mathrm{D}$ tube. Discretization: $Q_{1} / Q_{1}$ with a fissure. Velocity scale: from 0 up to 11.8 . For picture purposes, the mesh presented in this figure is quite coarse.
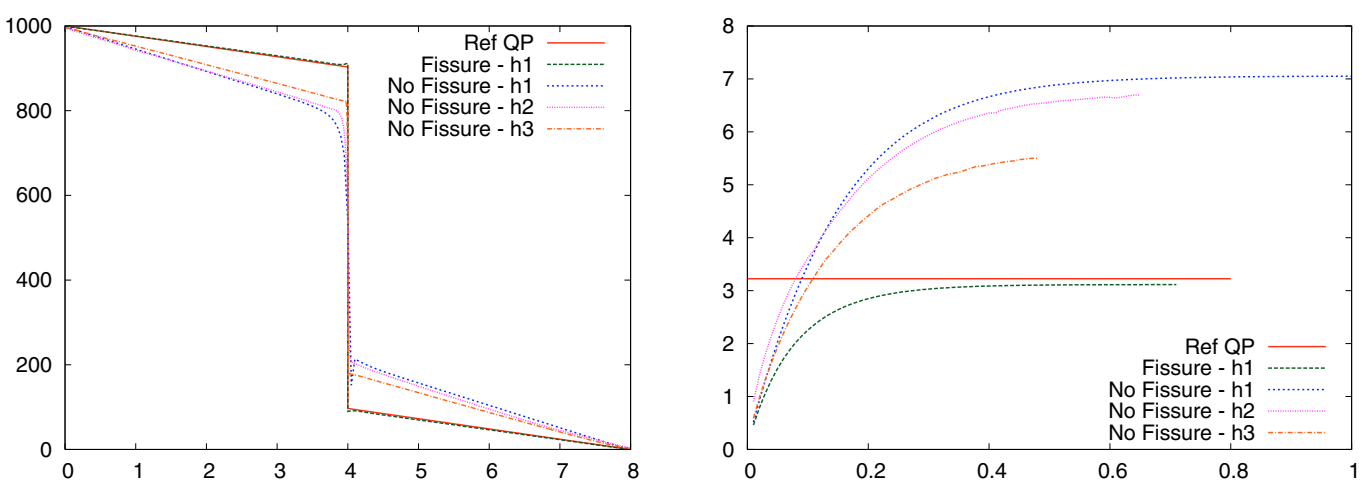

Figure 8. Tube 2d with a stent: influence of the mesh refinement on the solution, for the $Q_{1} / Q_{1}$ discretization $\left(r_{\gamma}=100\right)$. Left: pressure as a function of $x$. Right: out flux as a function of time. In the two figures, the first curve represents the "quasi-Poiseuille" flow approximation ("Ref $Q P$ "). The second curve represents the numerical solution on a coarse mesh with a discontinuous pressure approximation; the pressure is almost superimposed on the "Ref $Q P$ " curve. For the three remaining curves, the pressure was approximated continuously. When $h=h 1$ (second and third curve), the mesh is regular and contains 3200 cells. When $h=h 2$ (fourth curve) (resp. $h=h 3$ (fifth curve)), the mesh is locally refined around the interface and contains 6400 (resp. 12800 ) cells.

Figure 8 (right) and in Figure 9 (left), this simple formula is nevertheless useful to detect dramatically wrong solutions.

In conclusion, this test-case shows that it seems to be necessary to use a discontinuous approximation of the pressure across the stent, at least when realistic values of resistivity are used. Once a fissure is created on the stent, our stabilized $Q_{1} / Q_{1}$ method gives results which are similar to the stable $Q_{2} / P_{1}$ finite element. In the $3 \mathrm{D}$ simulations presented in the next section, we will limit ourselves to stabilized tetrahedral $P_{1} / P_{1}$ finite elements.

Remark 3.2. In the context of homogenization, Sánchez-Palencia [23] and Conca [10,11] studied the behavior of a flow through a planar sieve. Conca proved that the flow, away from the sieve, tends to satisfy a Stokes 

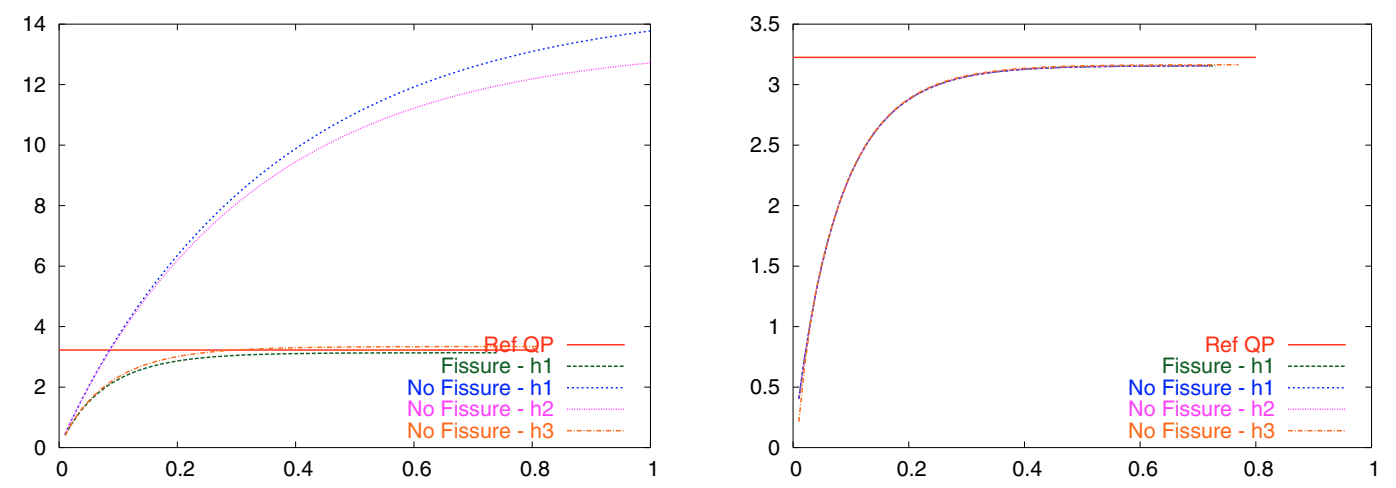

Figure 9. Tube 2d with a stent: influence of the mesh refinement on the solution, for the $Q_{2} / Q_{1}$ and the $Q_{2} / P_{1}$ discretizations. $r_{\gamma}=100$. Left and right: out flux as a function of time. Left: $Q_{2} / Q_{1}$ elements. Right: $Q_{2} / P 1$ elements. In the two figures, the first curve represents the "quasi-Poiseuille" flow approximation ("Ref $Q P$ "). When $h=h 1$ (second and third curves) (resp. $h=h 2$ (fourth curve)), the mesh is regular and contains 800 (resp. 3200) cells. When $h=h 3$ (fifth curve), the mesh is locally refined around the interface and still contains 3200 cells. Left: with no fissure at the interface, the pressure is approximated continuously, and one needs to refine the mesh a lot to obtain the correct solution. A good solution can be computed at a much cheaper cost with a fissured mesh. Right: all curves are superimposed. The pressure lies in a discontinuous space and therefore one can catch the discontinuity without extreme mesh refinement, nor a fissure in the mesh.

problem, with a constant velocity on the sieve. Some numerical solutions in the vicinity of the sieve were presented in [12]. Our numerical experiments show a very similar behavior (Fig. 7).

\subsubsection{Effect of a slanting interface}

We test in this section the behavior of a stent that is not orthogonal to the main direction of the flow. The domain is the same as in the previous section (see Fig. 6), except that the angle between the $O x$ axis and the interface $\gamma$ is no longer $\pi / 2$, but varies in $\{\pi / 3, \pi / 4, \pi / 6\}$. The computations were made with the same parameters as in the previous section, and using Q2/P1 finite elements. Different mesh refinements were used to ensure a good accuracy.

The results can be seen in Figure 10. Note that in the vicinity of the interface, the velocity is almost orthogonal to the stent. Far enough from the stent, the profile is approximately parabolic. Given a constant pressure drop, the more oblique the stent is, the less the flux is reduced by the resistivity (see Tab. 2).

\subsubsection{Effect of a non-planar interface}

We test in this section the behavior of a stent that is not planar. Once again, the domain is a 2D tube as in Section 3.2.1 (see Fig. 6), except that this time the stent has a parabolic shape (see Fig. 11). Parabola's equation is: $x-L_{1}+\beta\left(y^{2} / b^{2}-1\right)=0$, with a parameter $\beta$ varying $\in\{0, \pm 0.05, \pm 0.1, \pm 0.2, \pm 0.5, \pm 0.7, \pm 1\}$. Thus when $\beta=0$, the stent is planar as in Section 3.2.1, whereas when $\beta \neq 0$, the tip of the parabola is located at distance $\beta$ of the line $x=L_{1}$. The computations were made with the same parameters as in the previous section, and using Q2/P1 finite elements. Different mesh refinements were used to ensure a good accuracy.

The results can be seen in Figure 11. Note that in the vicinity of the interface, the velocity remains orthogonal to the stent. Far enough from the stent, the profile is approximately parabolic. Given a constant pressure drop, the greater the stent's curvature (i.e. the greater $\beta$ ), the less the flux is reduced by the resistivity (see Tab. 3). The flux value does not depend on the sign of $\beta$. 


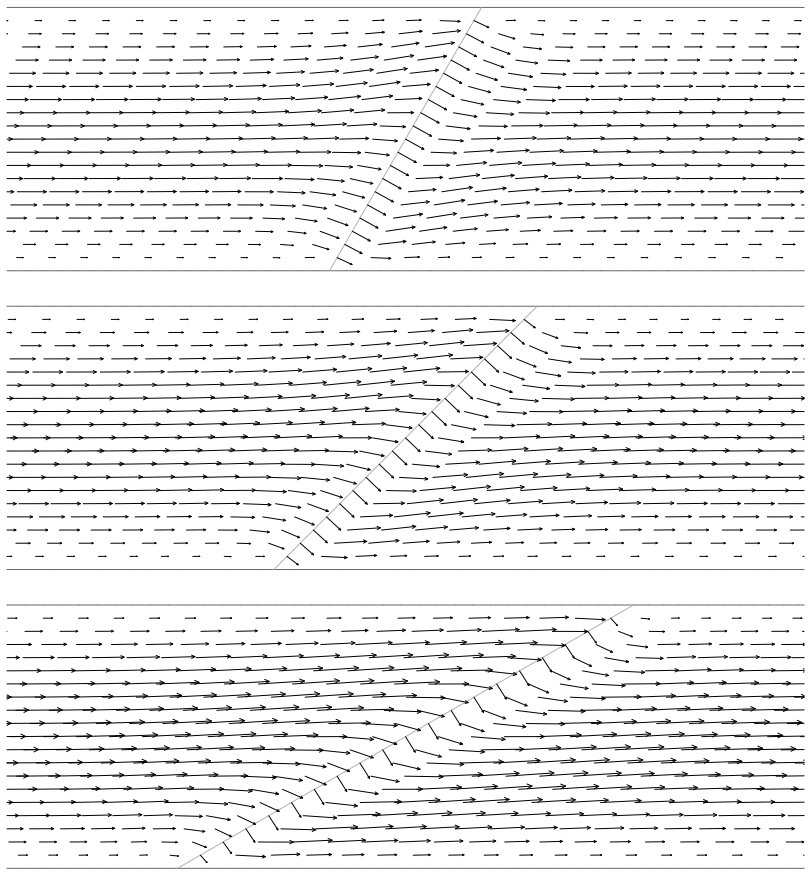

Figure 10. Tube $2 \mathrm{~d}$ with a slanting stent. Velocity with a resistive interface $\left(r_{\gamma}=100\right)$. From top to bottom: angle $\in\{\pi / 3, \pi / 4, \pi / 6\}$. For picture purposes, the mesh presented in this figure is quite coarse.

TABLE 2. Flux in the tube as a function of the angle between the axis of the flow and the interface $\gamma$. Resistivity $r_{\gamma}=100$. Imposed pressure drop $\Delta P=1000$.

\begin{tabular}{||c|c|c|c|c||}
\hline Angle & $\pi / 2$ & $\pi / 3$ & $\pi / 4$ & $\pi / 6$ \\
\hline Flux & 3.164 & 3.492 & 3.982 & 4.851 \\
\hline
\end{tabular}
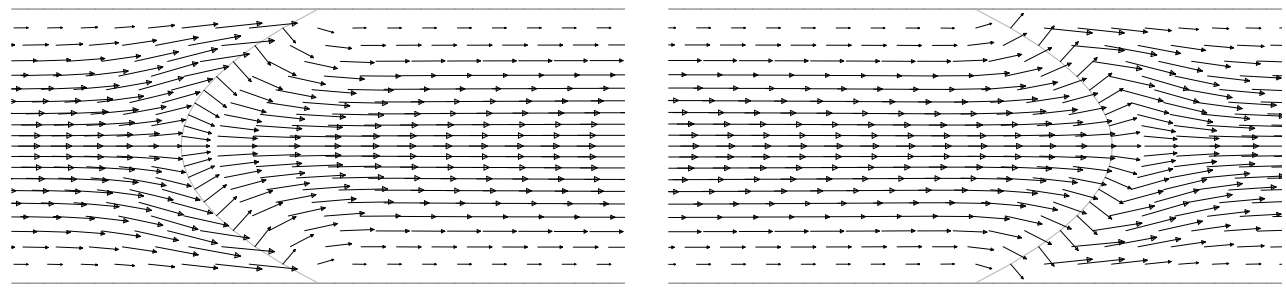

FiguRE 11. Tube $2 \mathrm{~d}$ with a parabolic stent. Velocity with a resistive interface $\left(r_{\gamma}=100\right)$. Left: $\beta=-0.2$, right $\beta=0.2$. For picture purposes, the mesh presented in this figure is quite coarse.

\subsubsection{An attempt at direct numerical simulation}

For the sake of comparison with the homogenized approach, we present some numerical simulations where the obstacles are directly taken into account. The domain is a 2D tube as in Section 3.2.1 (see Fig. 6), with $N_{t}$ small 1D obstacles of diameter $a_{\varepsilon}$ regularly spaced on $\gamma$. Except the viscosity that is taken $\mu=1$, all other 
TABLE 3. Flux in the tube as a function of the parameter $\beta$ of the parabola. Resistivity $r_{\gamma}=100$. Imposed pressure drop $\Delta P=1000$.

\begin{tabular}{||c|c|c|c|c|c|c|c||}
\hline$\beta$ & 0 & \pm 0.05 & \pm 0.1 & \pm 0.2 & \pm 0.5 & \pm 0.7 & \pm 1 \\
\hline Flux & 3.164 & 3.248 & 3.420 & 3.892 & 5.042 & 5.524 & 5.982 \\
\hline
\end{tabular}

TABLe 4. Flux in the tube and pressure drop across the stent as a function of the number of obstacles. A theoretical resistivity is given by the formula: $r_{\gamma}(\phi)=\Delta P * 2 b / \phi-\left(R_{1}+R_{2}\right)$, where $R_{i}=\frac{3 \mu L_{i}}{b^{2}}, i=1,2$. The theoretical limit given by homogenization is also given ("HOM."). Imposed pressure drop $\Delta P=1000, b=0.2 \mathrm{~cm}, L=4 \mathrm{~cm}$, viscosity $\mu=1$.

\begin{tabular}{||c|c|c|c|c|c||}
\hline$N_{t}$ & $\varepsilon$ & $a_{\varepsilon}$ & $\phi$ & $\Delta P^{\mathrm{St}}$ & $r_{\gamma}(\Phi)$ \\
\hline 16 & $1.25 E-2$ & $6.56 E-3$ & 0.334 & 512 & 598 \\
\hline 20 & $1 . E-2$ & $1.87 E-3$ & 0.412 & 397 & 370 \\
\hline 24 & $8.33 E-3$ & $5.31 E-4$ & 0.443 & 353 & 307 \\
\hline 28 & $7.14 E-3$ & $1.51 E-4$ & 0.450 & 342 & 289 \\
\hline \hline HOM. & 0 & 0 & 0.571 & 143 & 100 \\
\hline
\end{tabular}

parameters are the same as in Section 3.2.1. A no-slip condition $(\mathbf{u}=0)$ is imposed on the obstacles. Denote $\varepsilon=b / N_{t}$ the half space period. According to [1], $a_{\varepsilon}$ and $\varepsilon$ are related in two dimensions, as $\varepsilon$ tends to 0 , by

$$
-\varepsilon \log \left(a_{\varepsilon}\right)=C_{0} \quad \text { or } \quad a_{\varepsilon}=\exp \left(-C_{0} / \varepsilon\right) .
$$

In three dimensions, the relation would be $\frac{a_{\varepsilon}}{\varepsilon^{2}}=C_{0}$. Note that the size of the obstacle decreases very rapidly. To compare the limit problem and the obstacle problems, we consider a theoretical resistivity $r_{\gamma}=100$. We then determine $C_{0}$ and the variation of $a_{\varepsilon}=\exp \left(-2 \Pi /\left(r_{\gamma} \varepsilon\right)\right)$. The simulations have been performed with the $Q 2 / P 1$ finite element pair and the mesh has of course been refined around the obstacles. See the results in Table 4.

The behaviour of the solution is qualitatively correct since a stiff pressure drop does appear across the stent. We observe that the flux increases and the pressure drop decreases as $\varepsilon$ goes to 0 . Nevertheless, it would be necessary to still increase the number of obstacles to really illustrate that the solution of the direct simulations tends to the solution of the homogenized problem.

\subsection{Bifurcation in a realistic 3D geometry}

We present some results that are carried out on a realistic aneurysm geometry. Two cases are considered: a rigid arterial wall, or an elastic arterial wall. In all cases, the stent is considered rigid, and is represented by the model (1.1). The fluid-structure interaction between the blood and the artery that occurs when the arterial wall is compliant, is solved via a quasi-Newton algorithm, see [17].

\subsubsection{Mesh considerations}

The surface of a human cerebral artery bifurcation containing a huge terminal aneurysm (about $10 \mathrm{~mm}$ wide) was obtained by medical imaging techniques and meshed using Yams [15]. Then from the surface mesh a 3D mesh was created using GHS3D ${ }^{4}$. Some computations have already been achieved with this mesh $[18,22]$.

An idealized stent is inserted in the primary surface mesh, see Figure 12. For simplicity, we use a crude geometry for the stent: it is represented by three planar surfaces that intersect the entrance of the two branches

${ }^{4}$ See http://www-c.inria.fr/Eric.Saltel/gamma/ghs3d 

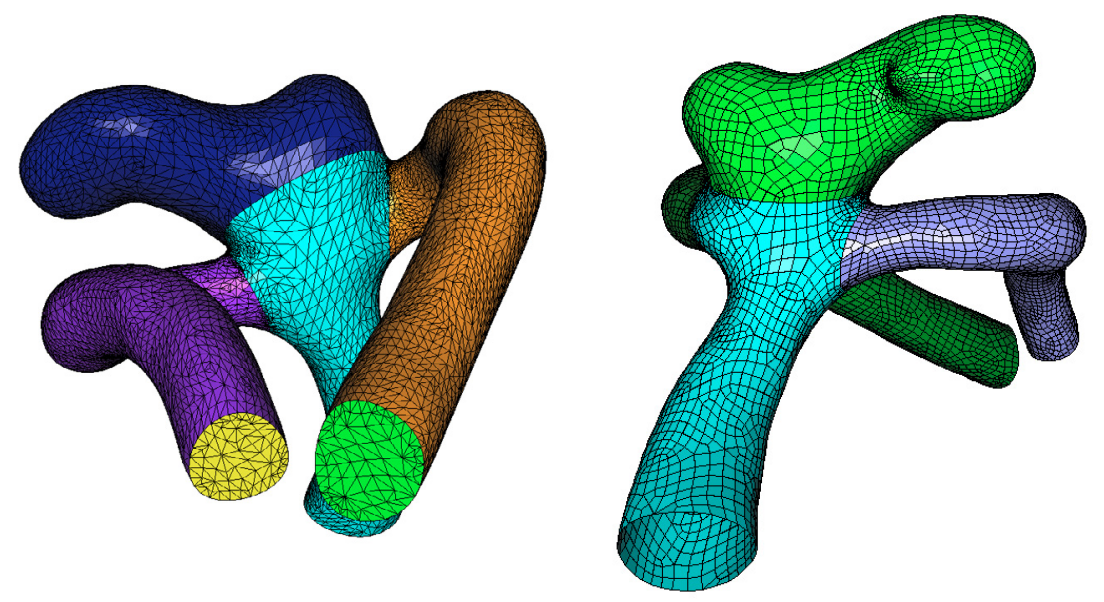

FiguRE 12. Mesh of the terminal aneurysm. Each subdomain is depicted with a different color. Left: fluid mesh; right: quadrangular shell mesh. Different view points. The stent separates the trunk artery (vertical inlet branch), from the two outlet branches, and from the aneurysm (top part). Visualization with Medit [16].
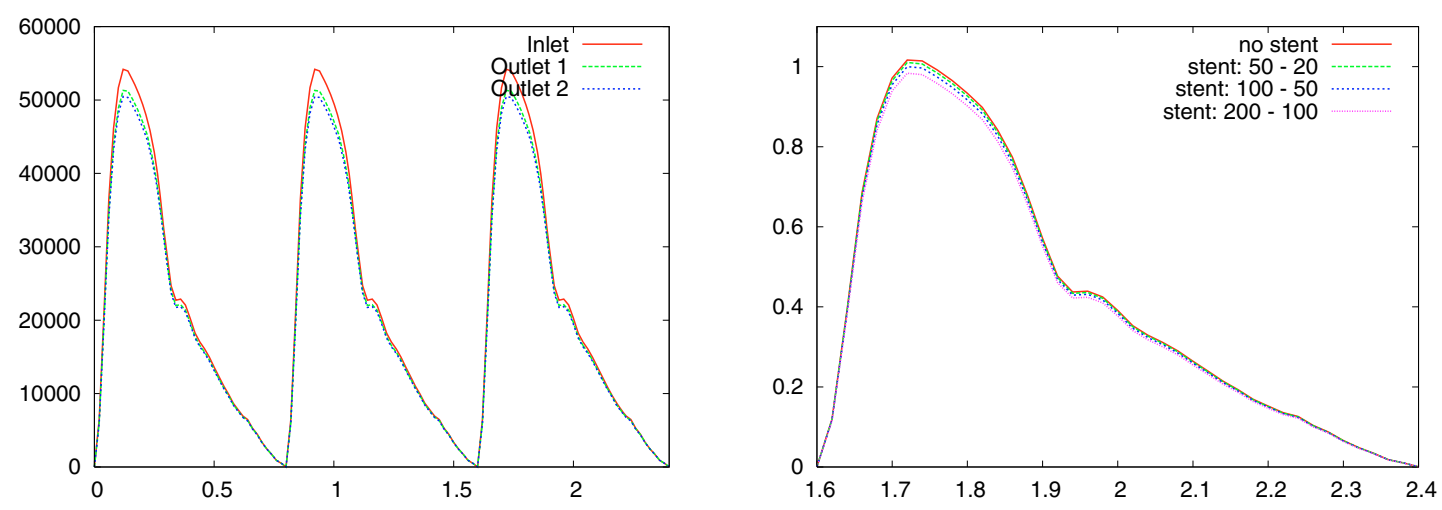

Figure 13. Left: input pressure in all test-cases (top curve), and output pressure in the test case 0 (no stent) at $\Gamma_{\text {out, } 1}$ (middle curve) and $\Gamma_{\text {out, } 2}$ (lowest curve). A slight asymmetry can be noticed. Scale: $[0 ; 6 E 4]$. Right: input flow (scale $[0 ; 1.1])$ as a function of time for one cardiac cycle. Curves, from top to bottom: test cases 0 (no stent), 4, 10, 13. The flow decreases very slowly when the resistivity increases (which is a desirable feature).

and of the aneurysm, thus decomposing the domain into four subdomains: the trunk of the artery called $\Omega_{\mathrm{tr}}$, the two branches $\Omega_{\mathrm{br} 1}$ and $\Omega_{\mathrm{br} 2}$ and the aneurysm $\Omega_{\mathrm{an}}$. Each subdomain surface is meshed in quadrangles using Yams, in order to use a shell structure code (based on Modulef ${ }^{5}$ ) for the arterial wall. Each subdomain is then meshed in tetrahedra in a conforming way using GHS3D, and glued together leaving a fissure at the interfaces. Finally, a 3D conforming mesh in tetrahedra with internal fissures representing the stent is created. We obtain also a quadrangular surface mesh whose vertices are the ones of the skin of the 3D mesh.

\footnotetext{
${ }^{5}$ See http://www-rocq.inria.fr/modulef/
} 


\subsubsection{Description of the problem}

Rigid wall. In the case of a rigid arterial wall, the computation is performed in different configurations over three cardiac cycles of period $T=0.8 \mathrm{~s}$. Problem (1.1) is completed with the following boundary conditions: we impose a pressure wave at the inlet $\Gamma_{\text {in }}$ (Neumann boundary conditions) and a flow-pressure relation simulating the rest of the arterial circuit is imposed at the outlets $\Gamma_{\text {out, } 1}$ and $\Gamma_{\text {out, } 2}$. On the rest of the boundary $\Gamma_{0}$, a no-slip condition is imposed:

$$
\begin{aligned}
\mathbf{T}(\mathbf{u}, p) \cdot \mathbf{n}\left(x, t^{n}\right) & =-p_{\text {in }}\left(t^{n}\right) & & \text { on } \Gamma_{\text {in }}, \\
\mathbf{T}(\mathbf{u}, p) \cdot \mathbf{n}\left(x, t^{n}\right) & =R_{\text {out }} \phi_{i}\left(t^{n}\right) & & \text { on } \Gamma_{\text {out }, \mathrm{i}}, i=1,2, \\
\mathbf{u} & =0 & & \text { on } \Gamma_{0},
\end{aligned}
$$

where $p_{\text {in }}$ is a given pressure function plotted in Figure $13, \phi_{i}=\int_{\Gamma_{\text {out }, \mathrm{i}}} \mathbf{u} \cdot \mathbf{n}$ is the out flux, and the resistivity $R_{\text {out }}$ was taken equal to $10^{5}$. The pressure varies over a $5.310^{4} \mathrm{~g} \cdot \mathrm{cm}^{-1} \cdot \mathrm{s}^{-2} \approx 40 \mathrm{~mm} \mathrm{Hg}$ amplitude, which corresponds to typical pressure drops in physiological conditions (between 80 and $120 \mathrm{~mm} \mathrm{Hg}$ ).

The viscosity is $\mu=0.035 \mathrm{~g} \cdot \mathrm{cm}^{-1} \cdot \mathrm{s}^{-1}$, the fluid density is $\rho_{f}=1 \mathrm{~g} \cdot \mathrm{cm}^{-3}$. The time step was taken equal to $0.01 \mathrm{~s}$. We used a Lagrange $P_{1} / P_{1}$ stabilized finite elements for the space discretization. Thanks to the fissures in the mesh, we let the pressure be discontinuous at the resistive interfaces, whereas the velocity is continuous.

Elastic wall. All the remarks made in the rigid wall case still apply, except that the computation is made over one cardiac cycle and a time step ten times smaller was taken $\left(\mathrm{d} t=10^{-3}\right)$.

Concerning the structure, a non-linear shell model is used, see for instance [6]. The Young modulus is $E=610^{6} \mathrm{dyn} \cdot \mathrm{cm}^{-2}$, the Poisson coefficient is $\nu=0.3$ and the thickness is $0.025 \mathrm{~cm}$ (about one tenth of the artery diameter). For simplicity, the shell is assumed to be clamped at its boundaries.

\subsubsection{Results}

In the different cases studied (see Tab. 5 for the rigid wall case and Tab. 6 for the moving wall case), we changed the resistivity of the stent, in order to assess the impact of the stent on the blood flow. We call $r_{\text {an }}$ the resistivity of the stent portion located at the entrance of the aneurysm, $r_{\mathrm{br}}$ the resistivity of the stent portion located at the entrance of the two branches. In the case 0 , there is no stent. In the other ones, the stent has a priori a non-homogeneous permeability: it is more resistive at the entrance of the aneurysm than at the entrance of the branches.

We present the solution at the systole in three main configurations: without any stent (test case 0), and when the aneurysm resistivity is twice as large as the ones at the entrance of the branches: stent with the resistivities $r_{\mathrm{an}}=50, r_{\mathrm{br}}=20$ (test case 4$)$, stent with $r_{\mathrm{an}}=100, r_{\mathrm{br}}=50$ (test case 10$)$. The results are summarized in Tables 5 and 6 .

In Figure 14, the peak pressure at the systole is depicted. It has to be noticed that the stent induces a pressure jump at the interfaces, notably between the trunk and the branches. When the arterial wall is compliant, the arteries and the aneurysm are slightly dilated under the pressure constraint, as expected. The pressure inside the aneurysm is not significantly modified by the stent in both the rigid and moving wall cases.

In Figure 15, the peak velocity along the trunk of the artery and inside the aneurysm is shown. Whereas the maximum velocity in the trunk is not really affected by a stent (it reaches its maximum value near the location where the trunk is the thinnest), the fluid velocity in the aneurysm is considerably reduced by the stent, as expected. A vortex in the aneurysm still exists for a resistivity $r_{\text {an }}=50$, but can hardly be seen. The velocity in the aneurysm is depicted in Figure 16: as expected, it decreases when the aneurysm resistivity $r_{\text {an }}$ increases (different scales in each picture), see also Tables 5 and 6: the ratio of maximum velocities in the aneurysm and in the trunk is reduced from $70 \%$ (no stent) to $23 \%$ when $r_{\text {an }}=50$, to $13 \%$ when $r_{\text {an }}=100$, and to $6 \%$ when $r_{\mathrm{an}}=200$. See also Figure 18, where the maximum velocity in the aneurysm as a function of time is plotted. The velocity in the aneurysm is slightly larger when the computation takes into account the compliance of the wall. 
TABLE 5. Different test-cases for the rigid arterial wall computations: stent resistivities in the aneurysm $r_{a n}$, and in the branches $r_{\mathrm{br}}\left(\mathrm{g} \cdot \mathrm{cm}^{-2} \cdot \mathrm{s}^{-1}\right)$, peak inflow $\left(\mathrm{cm}^{3} \cdot \mathrm{s}^{-1}\right)$, peak outflows at the two outlets, ratio of the maximum velocity in the aneurysm over the maximum velocity in the trunk, $L^{2}$ norm of the vorticity in the aneurysm and of the wall shear stress at the wall of the aneurysm.

\begin{tabular}{|c|c|c|c|c|c|c|c|c|}
\hline Case & $r_{\text {an }}$ & $r_{\mathrm{br}}$ & $\|\phi\|_{\Gamma_{\text {in }}, \infty}$ & $\left.\|\phi\|\right|_{\Gamma_{\text {out }, 1, \infty}}$ & $\|\phi\|_{\Gamma_{\text {out }, 2}, \infty}$ & $\frac{\|\mathbf{u}\|_{\Omega_{\text {an }}, \infty}}{\|\mathbf{u}\|_{0}}$ & $\|\nabla \times \mathbf{u}\|_{\Omega_{\mathrm{an}, 2}}$ & $\|W S S\|_{\partial \Omega_{\mathrm{an}, 2}}$ \\
\hline 0 & 0 & 0 & 1.02 & 0.513 & 0.504 & $69.9 \%$ & 61.7 & 11.6 \\
\hline 1 & 50 & 1 & 1.01 & 0.513 & 0.504 & $23.4 \%$ & 14.1 & 2.93 \\
\hline 2 & 50 & 5 & 1.01 & 0.513 & 0.503 & $23.3 \%$ & 14.0 & 2.90 \\
\hline 3 & 50 & 10 & 1.01 & 0.512 & 0.502 & $23.2 \%$ & 13.9 & 2.88 \\
\hline 4 & 50 & 20 & 1.01 & 0.51 & 0.5 & $23.0 \%$ & 13.7 & 2.82 \\
\hline 5 & 50 & 50 & 1.0 & 0.505 & 0.494 & $22.6 \%$ & 13.2 & 2.70 \\
\hline 6 & 100 & 1 & 1.02 & 0.513 & 0.504 & $12.7 \%$ & 7.3 & 1.61 \\
\hline 7 & 100 & 5 & 1.02 & 0.513 & 0.503 & $12.7 \%$ & 7.3 & 1.60 \\
\hline 8 & 100 & 10 & 1.01 & 0.512 & 0.502 & $12.7 \%$ & 7.2 & 1.59 \\
\hline 9 & 100 & 20 & 1.01 & 0.51 & 0.5 & $12.5 \%$ & 7.1 & 1.56 \\
\hline 10 & 100 & 50 & 1.0 & 0.506 & 0.495 & $12.2 \%$ & 6.9 & 1.49 \\
\hline 11 & 100 & 100 & 0.98 & 0.498 & 0.486 & $11.9 \%$ & 6.5 & 1.40 \\
\hline 12 & 200 & 50 & 1.0 & 0.506 & 0.495 & $6.5 \%$ & 3.5 & 0.80 \\
\hline 13 & 200 & 100 & 0.98 & 0.498 & 0.486 & $6.3 \%$ & 3.4 & 0.75 \\
\hline
\end{tabular}

TABLE 6. Different test-cases for the elastic arterial wall computations: stent resistivities in the aneurysm $r_{\mathrm{an}}$, and in the branches $r_{\mathrm{br}}\left(\mathrm{g} \cdot \mathrm{cm}^{-2} \cdot \mathrm{s}^{-1}\right)$, peak inflow $\left(\mathrm{cm}^{3} \cdot \mathrm{s}^{-1}\right)$, peak outflows at the two outlets, ratio of the maximum velocity in the aneurysm over the maximum velocity in the trunk, $L^{2}$ norm of the vorticity in the aneurysm and of the wall shear stress at the wall of the aneurysm.

\begin{tabular}{||c||c|c|c|c|c|c|c|c||}
\hline Case & $r_{\text {an }}$ & $r_{\text {br }}$ & $\|\phi\|_{\Gamma_{\text {in }, \infty}}$ & $\|\phi\|_{\Gamma_{\text {out }, 1}, \infty}$ & $\|\phi\|_{\Gamma_{\text {out }, 2, \infty}}$ & $\frac{\|\mathbf{u}\| \Omega_{\Omega_{\text {an }}, \infty}}{\| \mathbf{u}||_{\text {r }}, \infty}$ & $\|\nabla \times \mathbf{u}\|_{\Omega_{\text {an }, 2}}$ & $\|W S S\|_{\partial \Omega_{\text {an }}, 2}$ \\
\hline 0 & 0 & 0 & 1.05 & 0.523 & 0.513 & $73.8 \%$ & 71.8 & 12.9 \\
\hline 4 & 50 & 20 & 1.04 & 0.520 & 0.509 & $22.7 \%$ & 14.8 & 2.80 \\
\hline 10 & 100 & 50 & 1.03 & 0.516 & 0.503 & $12.9 \%$ & 7.6 & 1.47 \\
\hline
\end{tabular}

In Figure 17, the wall shear stress at the wall of the aneurysm is plotted. Two different scales are used, the one with no stent being five times larger than the other. The maximum values of the wall shear stress seem to appear at the same locations in all cases. No significant differences can be noticed between the rigid and moving wall computations.

As one would expect, when a given pressure drop is imposed, the flow in the artery slightly decreases as the stent resistivity of the branches increases. This is normal as the overall resistivity of the domain to the fluid is increased by the stent. Other numerical experiments show that this effect can be quite important, in particular when the pressure imposed $p_{\text {in }}$ is smaller ${ }^{6}$. One can note that the aneurysm stent has little influence over the

\footnotetext{
${ }^{6}$ In the same configuration, for a pressure $p_{\text {in }}$ about ten times smaller $(\approx 5 \mathrm{~mm} \mathrm{Hg})$, an outlet resistivity $R_{\text {out }}=1 E 4$ and a resistance $r_{\mathrm{br}}=50$, a flow reduction of about $10 \%$ was observed with the stent.
} 

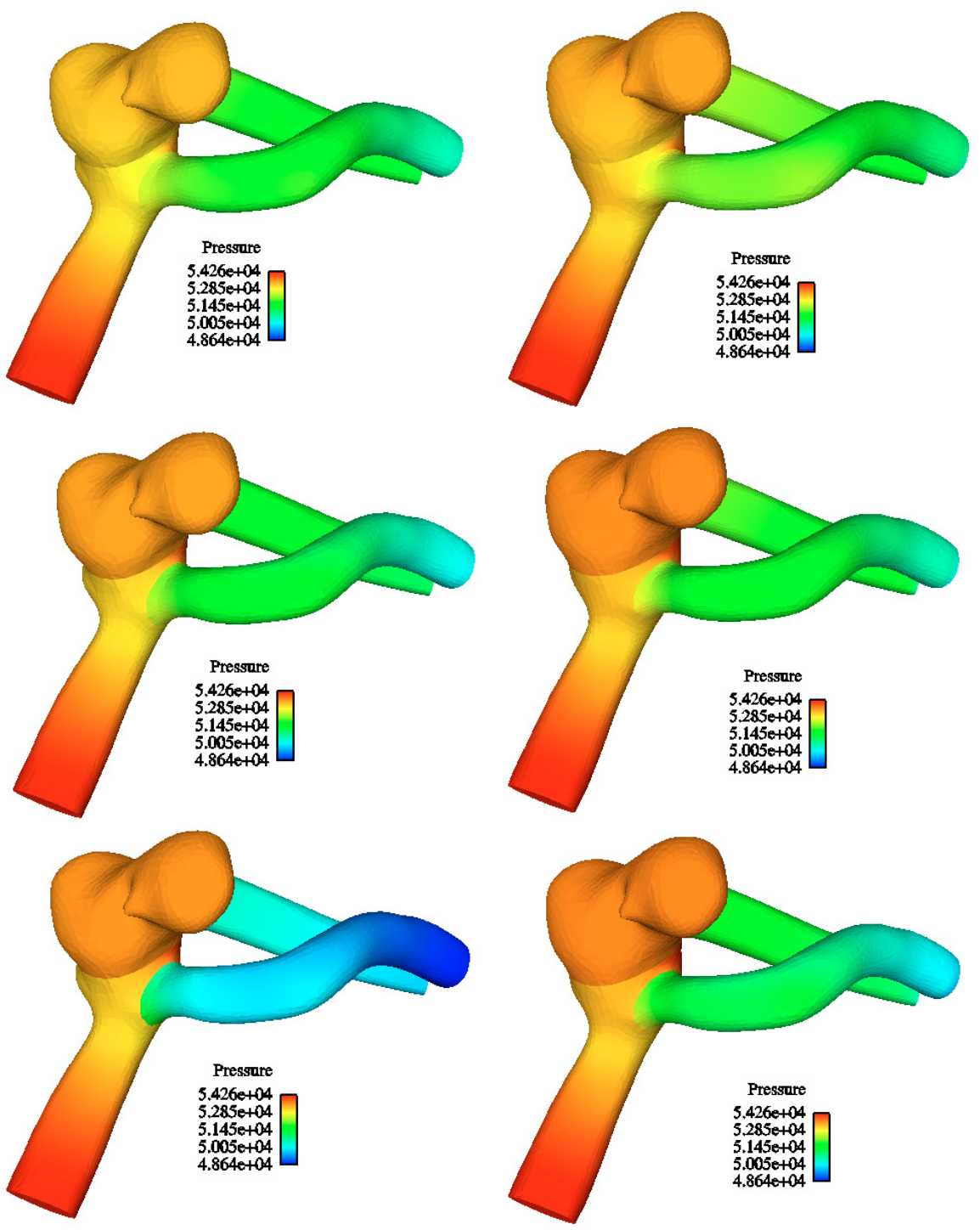

FiguRE 14. Systolic pressure for different resistivities, and with rigid or moving arterial walls. Scale: $\left[4.8 \times 10^{4}, 5.4 \times 10^{4}\right]$. Left column: rigid arterial wall. Right column: moving wall. From top to bottom: no stent, test case 4 (stent with the resistivities: $r_{\mathrm{an}}=50, r_{\mathrm{br}}=20$ ), test case $10\left(r_{\mathrm{an}}=100, r_{\mathrm{br}}=50\right)$.

global flow. In Figure 13, the input flow as a function of time is depicted over one period. We show in Figure 18 the $L^{2}$ norm of the wall shear stress on the wall of the aneurysm as a function of time. It is dramatically reduced by the stent. The vorticity inside the aneurysm shows a similar behavior. In Figure 19, we compared the maximum velocity in the aneurysm and the wall shear stress in the rigid and moving wall cases. There are no significant differences, except that, when the wall is compliant, the values are a bit larger. Finally, in Figure 20, the displacement of a point located on the topmost part of the aneurysm is depicted (for a moving 

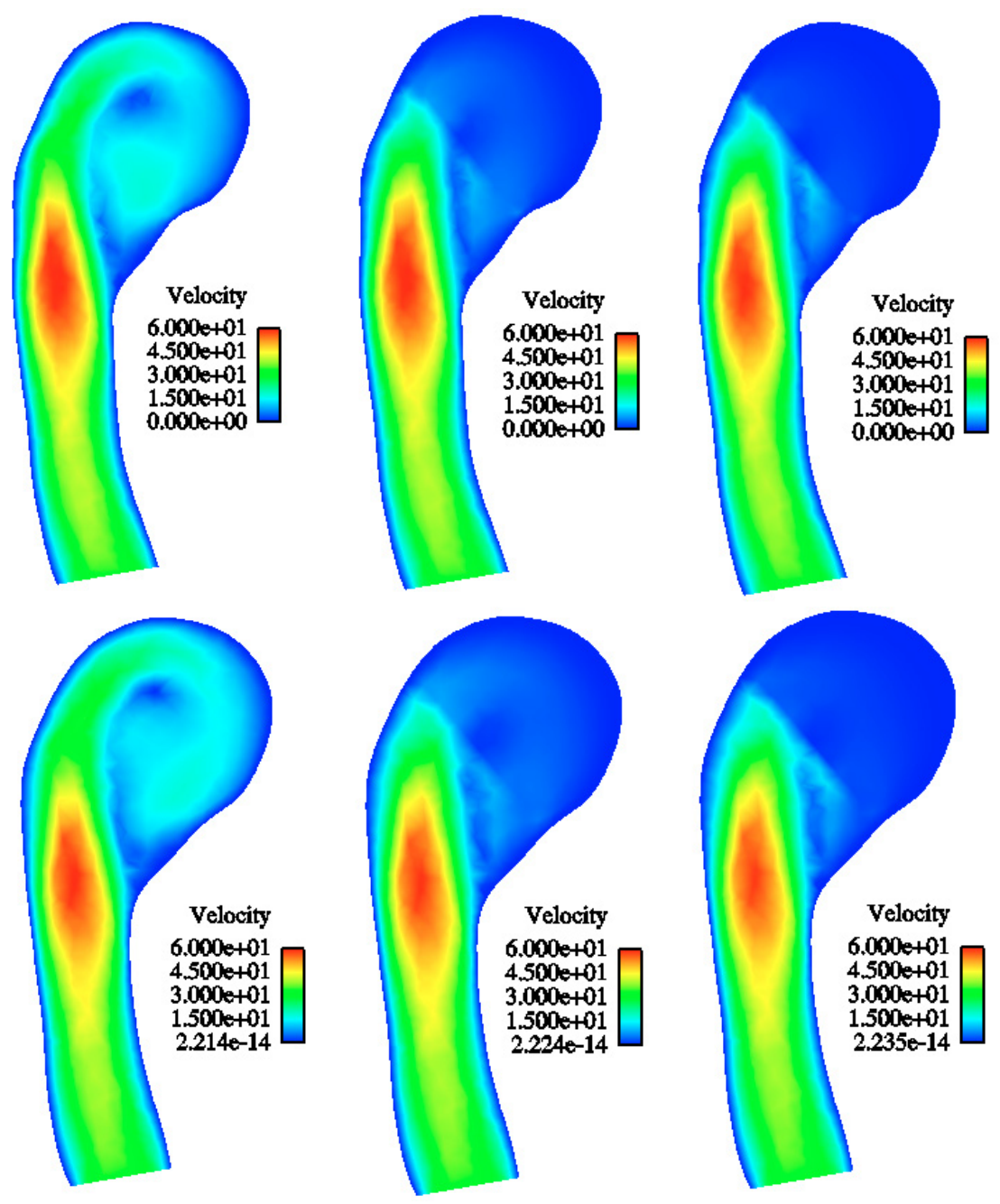

FIGURE 15. Systolic velocity in a planar section of the trunk and the aneurysm. The cut plane is located approximately along the trunk axis, and is perpendicular to the two entrances into the branches. The location of the aneurysm stent, if present, can be guessed as it limits the low velocity zone and the trunk region. Scale: [0,60]. Top: rigid arterial wall. Bottom: moving wall. From left to right: no stent, test cases $4\left(r_{\mathrm{an}}=50, r_{\mathrm{br}}=20\right), 10\left(r_{\mathrm{an}}=100, r_{\mathrm{br}}=50\right)$.

wall computation). When the stent is present $\left(r_{\mathrm{an}}=100\right)$, the resistivity induces a reduction of about $5 \%$ of the maximum displacement (see Tab. 7).

\subsubsection{Discussion}

The numerical experiment shows that the presence of a stent with some realistic resistivity properties considerably reduces the velocity in the aneurysm, and thus the vorticity and the wall shear stress on the wall of the aneurysm. Thus, one can predict that a blood clot could be created rapidly in the aneurysm, thanks to the stent. Besides, the global blood flow does not seem to be too much perturbed by the stent, out of the aneurysm: in particular, the outflow in physiological conditions remains almost unchanged. The results did not change 

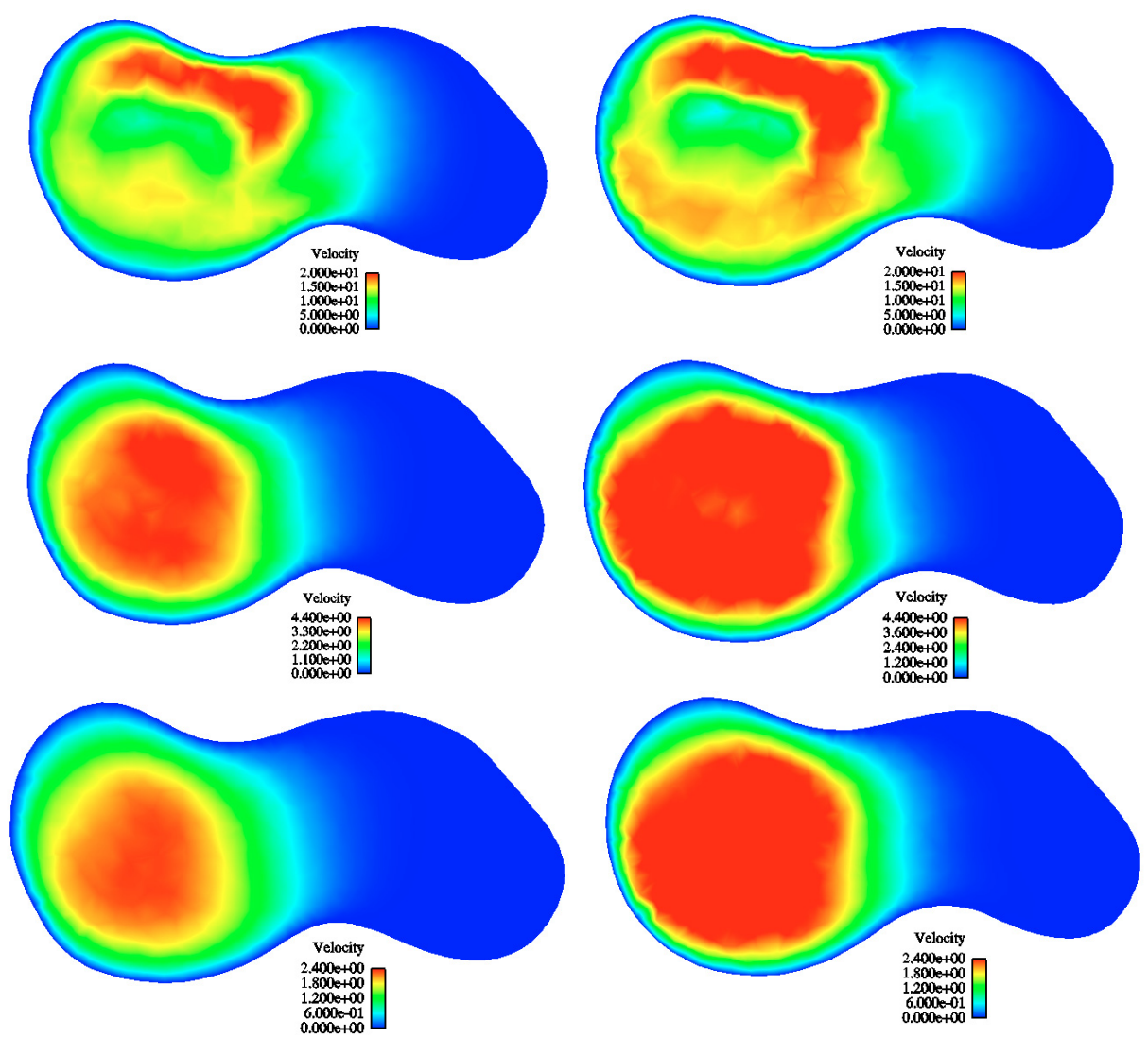

FiguRE 16. Systolic velocity in a planar section of the aneurysm. The cut plane is located approximately at the middle of the aneurysm, and is parallel to entrance of the aneurysm. Left column: rigid arterial wall. Right column: moving wall. From top to bottom: no stent (scale: $[0,20])$, test case $4\left(r_{\mathrm{an}}=50, r_{\mathrm{br}}=20\right.$, scale $\left.[0,4.4]\right)$, test case $10\left(r_{\mathrm{an}}=100, r_{\mathrm{br}}=50\right.$, scale: $[0,2.4])$. Note that the scales have been chosen to compare the simulations with and without elastic wall (left and right), but depend on the resistivity (from top to bottom) for the sake of clarity.

significantly between the rigid wall case and the moving wall case, thus, in this configuration, it does not seem necessary to perform the full fluid-structure interaction computation to obtain reasonable results.

As explained at the beginning of this paper, the proposed model has inherent limitations. Thus, the quantitative result provided in Tables 5 and 6 must be considered with care. Nevertheless, they indicate clear trends which may be useful. In addition, some of these trends were not expected, as the fact that the outflow is almost unaffected in the considered permeability range.

\section{Conclusions And Perspectives}

We have presented a model to represent the interaction between a stent and the blood flow in arteries. Our purpose was to build a model simple enough and easily parameterized with the available data. We have modelled the stent as a rigid dissipative interface which interacts with an incompressible fluid governed by the Navier-Stokes equations. This interface creates a jump of the normal stress that is proportional to the velocity. 

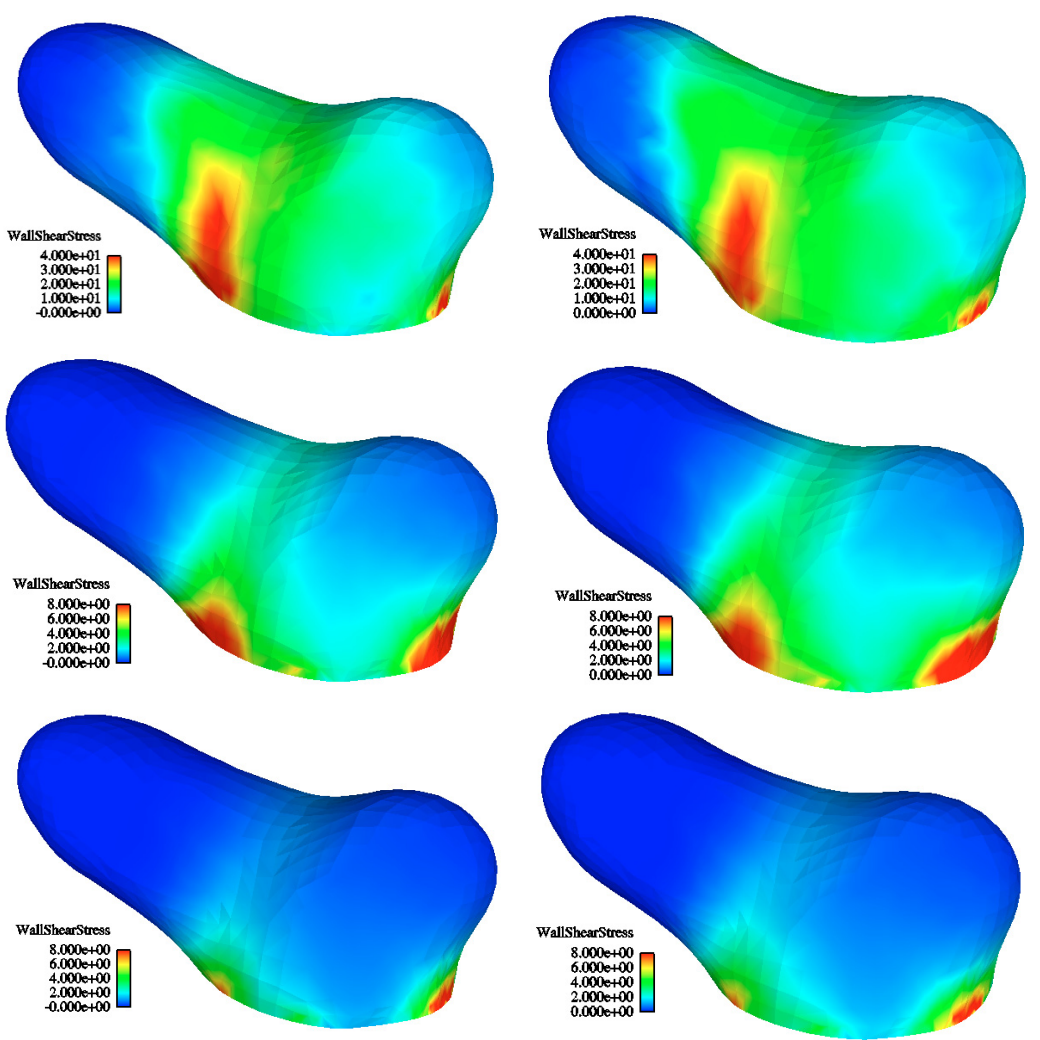

FIGURE 17. Systolic wall shear stress on the boundary of the aneurysm. Left column: rigid arterial wall. Right column: moving wall. From top to bottom: no stent (scale: [0,40]), test case $4\left(r_{\mathrm{an}}=50, r_{\mathrm{br}}=20\right.$, scale $\left.[0,8]\right)$, test case $10\left(r_{\mathrm{an}}=100, r_{\mathrm{br}}=50\right.$, scale: $\left.[0,8]\right)$. Note that the scales have been chosen to compare the simulations with and without elastic wall (left and right), but depend on the resistivity (from top to bottom) for the sake of clarity.
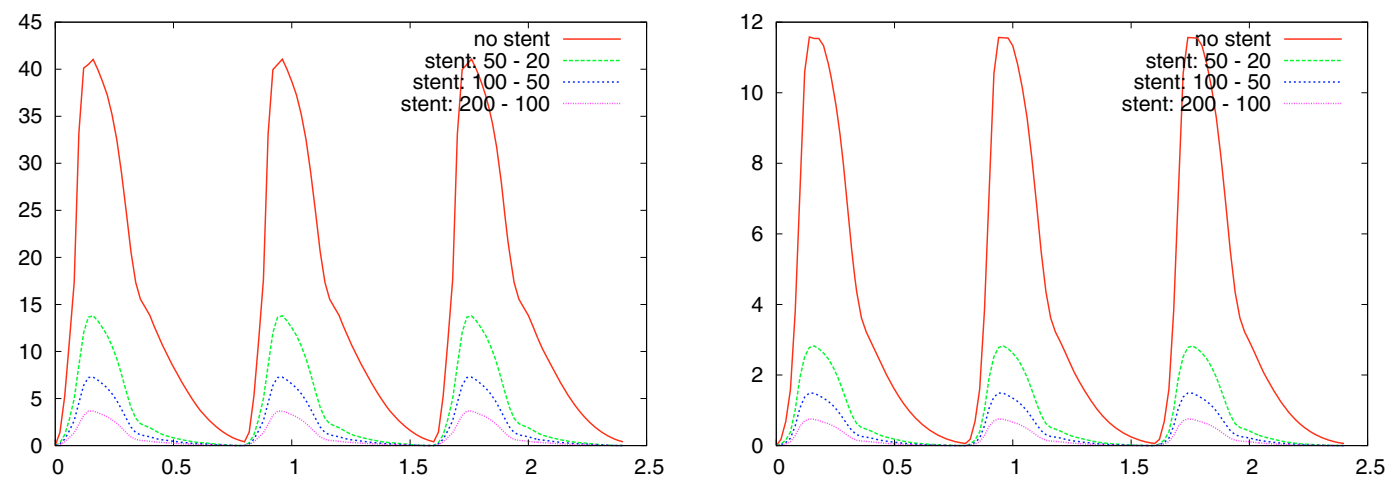

FIGURE 18. Rigid wall computation. Left: maximum velocity in the aneurysm (scale $[0 ; 45]$ ) as a function of time. Right: $L^{2}$ norm of the wall shear stress on the aneurysm wall (scale $[0 ; 12])$ as a function of time. Curves, from top to bottom: test cases 0 (no stent), 4, 10, 13. 

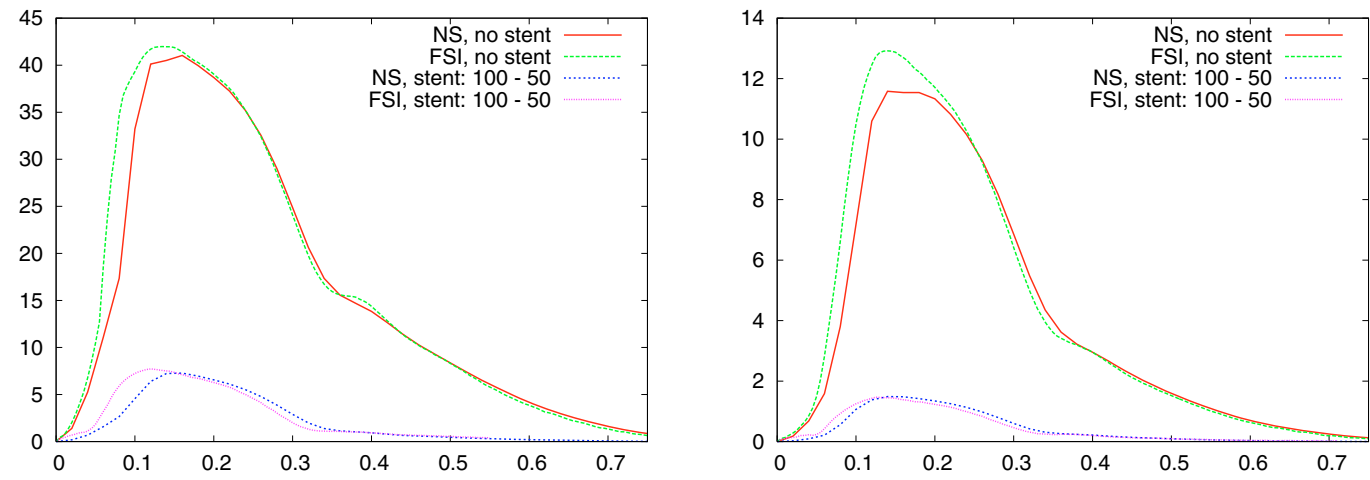

Figure 19. Comparison between rigid and moving arterial walls. Left: maximum velocity in the aneurysm (scale $[0 ; 45])$ as a function of time. Right: $L^{2}$ norm of the wall shear stress on the aneurysm wall (scale $[0 ; 14])$ as a function of time. Curves, from top to bottom: test cases 0 (no stent) with rigid ("NS") and moving wall ("FSI"), 10 with rigid and moving wall.

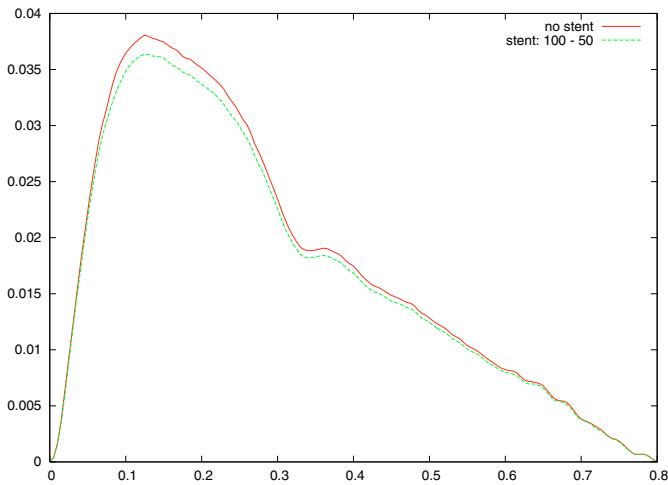

FIGURE 20. Displacement of a point located on top of the aneurysm as a function of time. Curves, from top to bottom: test cases 0 (no stent) and 10.

The proportionality coefficient is a resistivity that can be determined by measurements. The jump of normal stress induces a discontinuity of the pressure that has to be correctly approximated: the discrete pressure space must be discontinuous at the interface. A convergence analysis is presented in the stationary linear case for a stabilized finite element method, involving a discontinuous pressure at the interface. Some simple two dimensional numerical tests have confirmed the theoretical convergence rate. Other numerical experiments have been presented in a realistic three dimensional saccular terminal aneurysm. Given a fixed pressure drop imposed at the boundaries, the stent acts as a resistance that only slightly reduces the flow exiting the domain. The stent decreases the velocities and the vorticity in the aneurysm, and tends to reduce also the aneurysm wall stresses. This indicates that the process of thrombosis might occur in the aneurysm, as desired.

The work could be improved in several ways. First, more realistic stent geometries could used. Second, better outflow boundary conditions could be devised, using either an impedance [29] or a network of 1D models [14,21]. Finally, it would be interesting to take into account a model of coagulation in the aneurysm, following for instance [30]. 
TABLE 7. Effect of the resistivity on the wall displacement in different test-cases for the elastic arterial wall computations. Maximum displacement of a point located on top of the aneurysm.

\begin{tabular}{||c||c|c|c|c||}
\hline Case & $r_{\text {an }}$ & $r_{\text {br }}$ & max. disp. & disp. reduction \\
\hline 0 & 0 & 0 & $3.80 \times 10^{-2}$ & - \\
\hline 4 & 50 & 20 & $3.68 \times 10^{-2}$ & $3.2 \%$ \\
\hline 10 & 100 & 50 & $3.64 \times 10^{-2}$ & $4.4 \%$ \\
\hline
\end{tabular}

Acknowledgements. This work was partially supported by Cardiatis. The authors thank N. Frid and P. Gruffaz (Cardiatis) who proposed this study and whose encouragements and constructive suggestions were of great help.

\section{REFERENCES}

[1] G. Allaire, Homogenization of the Navier-Stokes equations in open sets perforated with tiny holes. II. Noncritical sizes of the holes for a volume distribution and a surface distribution of holes. Arch. Rational Mech. Anal. 113 (1991) 261-298.

[2] J.L. Berry, A. Santamarina, J.E. Jr. Moore, S. Roychowdhury and W.D. Routh, Experimental and computational flow evaluation of coronary stents. Ann. Biomed. Eng. 28 (2000) 386-398.

[3] F. Brezzi and M. Fortin, Mixed and Hybrid Finite Element Methods. Springer-Verlag, Berlin (1991).

[4] A. Brillard, Asymptotic flow of a viscous and incompressible fluid through a plane sieve, in Progress in partial differential equations: calculus of variations, applications (Pont-à-Mousson, 1991), Pitman Res. Notes Math. Ser. 267, Longman Sci. Tech., Harlow (1992) 158-172.

[5] E. Burman and P. Hansbo, Edge stabilization for the generalized Stokes problem: a continuous interior penalty method. Comput. Methods Appl. Mech. Engrg. 195 (2006) 2393-2410.

[6] D. Chapelle and K.J. Bathe, The finite element analysis of shell - Fundamentals. Springer-Verlag (2004).

[7] P.G. Ciarlet, The finite element method for elliptic problems, Classics in Applied Mathematics 40. Society for Industrial and Applied Mathematics (SIAM), Philadelphia, PA (2002). Reprint of the 1978 original [North-Holland, Amsterdam; MR0520174 $(58 \# 25001)]$.

[8] P. Clément, Approximation by finite element functions using local regularization. Rev. Française Automat. Informat. Recherche Opérationnelle Sér. Rouge Anal. Numér. 9(R-2) (1975) 77-84.

[9] R. Codina and J. Blasco, A finite element formulation for the Stokes problem allowing equal velocity-pressure interpolation. Comput. Methods Appl. Mech. Engrg. 143 (1997) 373-391.

[10] C. Conca, Étude d'un fluide traversant une paroi perforée, I. Comportement limite près de la paroi. J. Math. Pures Appl. 66 (1987) $1-43$.

[11] C. Conca, Étude d'un fluide traversant une paroi perforée, II. Comportement limite loin de la paroi. J. Math. Pures Appl. 66 (1987) 45-70.

[12] C. Conca and M. Sepúlveda, Numerical results in the Stokes sieve problem. Rev. Internac. Métod. Numér. Cálc. Diseñ. Ingr. 5 (1989) 435-452.

[13] A. Ern and J.-L. Guermond, Theory and practice of finite elements, Applied Mathematical Sciences 159. Springer-Verlag, New York (2004)

[14] L. Formaggia, J.-F. Gerbeau, F. Nobile and A. Quarteroni, On the coupling of 3D and 1D Navier-Stokes equations for flow problems in compliant vessels. Comput. Methods Appl. Mech. Engrg. 191 (2001) 561-582.

[15] P. Frey, Yams: A fully automatic adaptive isotropic surface remeshing procedure. Technical report 0252, INRIA, Rocquencourt, France, Nov. (2001).

[16] P. Frey, Medit: An interactive mesh visualisation software. Technical report 0253, INRIA, Rocquencourt, France, Dec. (2001).

[17] J.-F. Gerbeau and M. Vidrascu, A quasi-Newton algorithm based on a reduced model for fluid structure problems in blood flows. ESAIM: M2AN 37 (2003) 631-647.

[18] J.-F. Gerbeau, M. Vidrascu and P. Frey, Fluid-structure interaction in blood flows on geometries coming from medical imaging. Comput. Struct. 83 (2005) 155-165.

[19] V. Girault and P.-A. Raviart, Finite element methods for Navier-Stokes equations - Theory and algorithms, Springer Series in Computational Mathematics 5. Springer-Verlag, Berlin (1986).

[20] T.J.R. Hughes, L.P. Franca and M. Balestra, A new finite element formulation for computational fluid dynamics: V. Circumventing the Babuska-Brezzi condition: a stable Petrov-Galerkin formulation of the Stokes problem accommodating equal-order interpolations. Comp. Meth. App. Mech. Eng. 59 (1986) 85-99.

[21] A. Quarteroni and L. Formaggia, Mathematical modelling and numerical simulation of the cardiovascular system, in Handbook of Numerical Analysis XII, North-Holland, Amsterdam (2004) 3-127. 
[22] S. Salmon, M. Thiriet and J.-F. Gerbeau, Medical image-based computational model of pulsatile flow in saccular aneurisms. ESAIM: M2AN 37 (2003) 663-679.

[23] E. Sánchez-Palencia, Problèmes mathématiques liés à l'écoulement d'un fluide visqueux à travers une grille, in Ennio De Giorgi colloquium (Paris, 1983), Res. Notes in Math. 125, Pitman, Boston, USA (1985) 126-138.

[24] L.R. Scott and S. Zhang, Finite element interpolation of nonsmooth functions satisfying boundary conditions. Math. Comp. 54(190) (1990) 483-493.

[25] D.A. Steinman, J.S. Milner, C.J. Norley, S.P. Lownie and D.W. Holdsworth, Image-based computational simulation of flow dynamics int a giant intracranial aneurysms. Am. J. Neuroradiol. 24 (2003) 559-566.

[26] G.R. Stuhne and D.A. Steinman, Finite-element modeling of the hemodynamics of stented aneurysms. J. Biomech. Eng. 126 (2004) 382-387.

[27] V. Thomée, Galerkin finite element methods for parabolic problems, Springer Series in Computational Mathematics 25. Springer-Verlag, Berlin, second edition (2006).

[28] L. Tobiska and V. Verfurth, Analysis of a streamline diffusion finite element method for the Stokes and Navier-Stokes equations. SIAM J. Numer. Anal. 33 (1996) 107-127.

[29] I.E. Vignon-Clementel, C.A. Figueroa, K.E. Jansen and C.A. Taylor, Outflow boundary conditions for three-dimensional finite element modeling of blood flow and pressure in arteries. Comput. Methods Appl. Mech. Engrg. 195 (2006) 3776-3796.

[30] N.T. Wang and A.L. Fogelson, Computational methods for continuum models of platelet aggregation. J. Comput. Phys. 151 (1999) 649-675. 